\title{
VOLUME OF HYPERCUBES CLIPPED BY HYPERPLANES AND COMBINATORIAL IDENTITIES*
}

\author{
YUNHI $\mathrm{CHO}^{\dagger}$ AND SEONHWA $\mathrm{KIM}^{\ddagger}$
}

\begin{abstract}
There is an elegant expression for the volume of hypercube $[0,1]^{n}$ clipped by a single hyperplane. In the article, the formula is generalized to the case of more than one hyperplane. An important foundation for the result is Lawrence's formula and a way to weaken two restrictions of simplicity and non-parallelness in his formula is also considered. Several concrete volume formulas of clipped hypercubes are derived explicitly and the corresponding combinatorial identities are obtained as an application.
\end{abstract}

Key words. Volume, Hypercube, Combinatorial identity.

AMS subject classifications. 52A38, 90C57.

1. Introduction. A unit hypercube is a convex polytope defined by $[0,1]^{n}$ in $\mathbb{R}^{n}$. It may be a very basic geometric object and the simplest convex polytope, but it still has interesting unsolved questions (for example, see [14]). It has turned out that the computation of the volume of convex polytopes is algorithmically hard [4] since it usually requires difficult work like vertex/facet enumeration, even for the case of hypercubes clipped by only one hyperplane [7]. For these reasons, both numerical approximations and exact calculations have been extensively studied from an algorithmic point of view.

In this paper, we will only focus on closed and concrete formulas using matrix computations. Although this doesn't require heavy machinery (but is technically complicated), the resulting volume formula is fairly concrete and hence has some applications like producing a certain class of combinatorial identities. Furthermore, it would be interesting to investigate the relation between our formulation focused on $[0,1]^{n}$ and Filliman's study [5, Section 3].

For the easiest case of a hypercube clipped by only one hyperplane, there is an interesting simple formula giving the volume as the following. The notation $\left|0_{\mathbf{v}}\right|$ for a vector $\mathbf{v} \in \mathbb{R}^{n}$ indicates the number of zeros in the entries and $F^{0}$ denotes the set of vertices of $[0,1]^{n}$. The half space $H_{1}^{+}$is given by

$$
\left\{\mathbf{x} \mid g_{1}(\mathbf{x}):=\mathbf{a} \cdot \mathbf{x}+r_{1}=a_{1} x_{1}+a_{2} x_{2}+\cdots+a_{n} x_{n}+r_{1} \geq 0\right\}
$$

with $\prod_{t=1}^{n} a_{t} \neq 0$. Then we have:

THEOREM 1.1.

$$
\operatorname{vol}\left([0,1]^{n} \cap H_{1}^{+}\right)=\sum_{\mathbf{v} \in F^{0} \cap H_{1}^{+}} \frac{(-1)^{\left|0_{\mathbf{v}}\right|} g_{1}(\mathbf{v})^{n}}{n ! \prod_{t=1}^{n} a_{t}} .
$$

${ }^{*}$ Received by the editors on February 18, 2017. Accepted for publication on March 26, 2020. Handling Editor: Bryan L. Shader. Corresponding Author: Seonhwa Kim.

$\dagger$ Department of Mathematics, University of Seoul, Seoul 02504, Korea (yhcho@uos.ac.kr). Supported by the 2019 Research Fund of University of Seoul.

${ }^{\ddagger}$ School of Mathematics, Korea Institute for Advanced Study, Seoul 02455, Korea (seonhwa17kim@kias.re.kr, seonhwa17kim@gmail.com). Supported by the National Research Foundation of Korea (NRF) grant funded by the Korea government (MSIT) (no. 2019R1C1C1003383), and supported by the Institute for Basic Science (IBS-R003-D1). 
This formula seems to have first appeared in [1], but a very similar idea seems to go back much earlier [12]. Although it has been revisited several times (for example, see Section 2 in [11]), as far as the authors know, a volume formula for the case of more than one hyperplane had not been seriously studied yet. We generalize this formula to the case of an arbitrary number of hyperplanes on the basis of Lawrence's work $[9]$.

Our volume formulas have concrete and explicit expressions, which have some benefits for the case of a small number of hyperplanes but a large dimensional cube. One can use our formula even for the case of sufficiently many hyperplanes making up a fully general polytope. But the greater the number of hyperplanes, the less useful our formula seems to be, because the characteristics coming from the shape of cube tend to disappear and the formula essentially becomes almost the same as Lawrence's.

The general formulas will be described in Section 4.3. Before doing that, let's look at two hyperplanes, which is a corollary of Theorem 4.6 (the detailed description is given in Section 5.1). Let the half spaces $H_{1}^{+}$ and $H_{2}^{+}$be given by

$$
\begin{aligned}
& H_{1}^{+}=\left\{\mathbf{x} \mid g_{1}(\mathbf{x}):=a_{1} x_{1}+a_{2} x_{2}+\cdots+a_{n} x_{n}+r_{1} \geq 0\right\} \text { and } \\
& H_{2}^{+}=\left\{\mathbf{x} \mid g_{2}(\mathbf{x}):=b_{1} x_{1}+b_{2} x_{2}+\cdots+b_{n} x_{n}+r_{2} \geq 0\right\}
\end{aligned}
$$

with good clipping conditions. See Section 2 for the notation and Section 4.2 for the definition of good clipping conditions. Then Theorem 1.1 is generalized to the case of two hyperplanes as follows.

Corollary 1.2 .

$$
\begin{aligned}
\operatorname{vol}\left([0,1]^{n} \cap H_{1}^{+} \cap H_{2}^{+}\right)= & \sum_{\mathbf{v} \in F^{0} \cap H_{1}^{+} \cap H_{2}^{+}} \frac{(-1)^{\left|0_{\mathbf{v}}\right|} g_{2}(\mathbf{v})^{n}}{n ! \prod_{t=1}^{n} b_{t}} \\
& -\sum_{\mathbf{v} \in F^{1} \cap H_{1} \cap H_{2}^{+}} \frac{(-1)^{\left|0_{\mathbf{v}}\right|} a_{*(\mathbf{v})}^{n} g_{2}(\mathbf{v})^{n}}{n !\left|a_{*(\mathbf{v})}\right| b_{*(\mathbf{v})} \prod_{t \in[n] \backslash *(\mathbf{v})} \mid \begin{array}{cc}
a_{*(\mathbf{v})} & b_{*(\mathbf{v})} \\
a_{t} & b_{t} \mid
\end{array} .} .
\end{aligned}
$$

Interestingly, volumes of hypercubes clipped by various choices of hyperplanes produce non-trivial combinatorial identities. Let us see several examples.

THEOREM 1.3. For arbitrary $y \in \mathbb{R}, a_{1}, a_{2}, \ldots, a_{n} \in \mathbb{R}$ and an integer $n \geq 0$,

$$
y^{n}+\sum_{i=1}^{n} \sum_{1 \leq t_{1}<t_{2}<\cdots<t_{i} \leq n}(-1)^{i}\left(y+a_{t_{1}}+\cdots+a_{t_{i}}\right)^{n}=(-1)^{n} n ! a_{1} a_{2} \cdots a_{n},
$$

or equivalently,

$$
\sum_{i=1}^{n} \sum_{1 \leq t_{1}<t_{2}<\cdots<t_{i} \leq n}(-1)^{i}\left(a_{t_{1}}+\cdots+a_{t_{i}}\right)^{k}= \begin{cases}-1, & \text { if } k=0 \\ 0, & \text { if } k=1,2, \ldots, n-1 \\ (-1)^{n} n ! a_{1} a_{2} \cdots a_{n}, & \text { if } k=n\end{cases}
$$

This is related to an old Prouhet-Tarry-Escott problem [10] and there are many not so difficult proofs. But it can be proved by Theorem 1.1 and we think the geometric proof using a clipped cube is a new approach. 
The next formula also may be obvious to someone familiar with Vandermonde matrices or Lagrange's interpolation formula. However, it can also be proved directly by Corollary 1.2 whose geometric description is a simplex clipped by one hyperplane (see [2]) or the hypercube $[0,1]^{n}$ clipped by two hyperplanes: a general hyperplane and a special hyperplane which passes through all standard basis vectors.

TheOREM 1.4. For arbitrary $y \in \mathbb{R}$, distinct non-zero $a_{1}, a_{2}, \ldots, a_{n} \in \mathbb{R}$ and an integer $n \geq 0$,

$$
\frac{y^{n}}{a_{1} a_{2} \cdots a_{n}}-\sum_{i=1}^{n} \frac{\left(y+a_{i}\right)^{n}}{a_{i}\left(a_{1}-a_{i}\right)\left(a_{2}-a_{i}\right) \cdots\left(\widehat{a_{i}-a_{i}}\right) \cdots\left(a_{n}-a_{i}\right)}=(-1)^{n}
$$

or equivalently,

$$
\sum_{i=1}^{n} \frac{a_{i}^{k}}{\left(a_{1}-a_{i}\right)\left(a_{2}-a_{i}\right) \cdots\left(\widehat{a_{i}-a_{i}}\right) \cdots\left(a_{n}-a_{i}\right)}= \begin{cases}\frac{1}{a_{1} a_{2} \cdots a_{n}}, & \text { if } k=-1, \\ 0, & \text { if } k=0,1, \ldots, n-2, \\ (-1)^{n-1}, & \text { if } k=n-1,\end{cases}
$$

where "^" means omitting the term.

Furthermore, if we take $a_{1}=a_{2}=\cdots=a_{n}=1$ in Theorem 1.3, then we get the following corollary which is a studied form in combinatorial enumeration (for example, see [13]).

COROLlaRY 1.5. For arbitrary $y \in \mathbb{R}$ and an integer $n \geq 0$,

$$
\sum_{i=0}^{n}(-1)^{i}\left(\begin{array}{c}
n \\
i
\end{array}\right)(y+i)^{n}=(-1)^{n} n !
$$

or equivalently,

$$
\sum_{i=0}^{n}(-1)^{i}\left(\begin{array}{c}
n \\
i
\end{array}\right) i^{k}= \begin{cases}0, & \text { if } k=0,1, \ldots, n-1, \\
(-1)^{n} n !, & \text { if } k=n .\end{cases}
$$

Interestingly, all the above identities are unified under one umbrella via a volume expression for a particular clipped hypercube. Before showing this, we introduce the following set-theoretic notation for the sake of convenience,

$$
\begin{gathered}
A:=\left\{a_{1}, a_{2}, \ldots, a_{n}\right\}, \quad\|A\|:=\sum_{a \in A} a, \quad A !:=\prod_{a \in A} a, \\
R_{A}(a):=\prod_{b \in A \backslash a} \frac{b}{b-a}, \quad R_{A}(I):=\sum_{a \in I} R_{A}(a) .
\end{gathered}
$$

Then Theorem 1.3 and Theorem 1.4 are written in an economic way as follows.

$$
\begin{aligned}
& \sum_{I \subset A}(-1)^{|I|}\|I\|^{k}= \begin{cases}0, & \text { if } k=0,1, \ldots, n-1, \\
(-1)^{n} n ! A !, & \text { if } k=n .\end{cases} \\
& \sum_{a \in A} R_{A}(a) a^{k}= \begin{cases}1, & \text { if } k=0, \\
0, & \text { if } k=1,2, \ldots, n-1, \\
(-1)^{n-1} A !, & \text { if } k=n .\end{cases}
\end{aligned}
$$


We can also obtain the following identity which can be derived from Corollary 1.2 (for the proof, see Theorem 6.4).

TheOREm 1.6. For $A=\left\{a_{1}, \ldots, a_{n}\right\}$ and an integer $l=1, \ldots, n$,

$$
\sum_{\substack{I \subset A \\
|I|<l}}(-1)^{|I|}\|I\|^{k}+\sum_{\substack{I \subset A \\
|I|=l}}(-1)^{l}\|I\|^{k} R_{A}(I)= \begin{cases}0, & \text { if } k=0,1, \ldots, n-1 \\
A ! \sum_{i=0}^{l-1}(-1)^{n-i}\left(\begin{array}{c}
n \\
i
\end{array}\right)(l-i)^{n}, & \text { if } k=n .\end{cases}
$$

The identity in Theorem 1.6 itself may already be known, but the proof using the volume of clipped hypercubes seems new. Finally, we would like to remark that the above identities are all symmetric functions. In Section 6 and the Appendix, we will give several identities, some symmetric and others not.

Let us outline our article. We will introduce notation in Section 2, and review and reorganize Lawrence's formula and explain our $\epsilon$-perturbation method in Section 3. The statements of main theorems and proofs will be given in Section 4. Several concrete examples will be presented with more explicit expressions in Section 5. In the final Section 6, we will derive a family of combinatorial identities using the volume of clipped hypercubes.

2. Notation. In this paper, the letters $n$ and $m$ correspond to the dimension of $\mathbb{R}^{n}$ and the number of hyperplanes respectively unless otherwise specified. A single bold letter always denotes a vector in $\mathbb{R}^{n}$ like $\mathbf{x}=\left(x_{1}, \ldots, x_{n}\right)$ and we abuse notation for column vectors and row vectors if it is not confusing. Let $\mathbf{e}_{i}$ denote the $i$-th vector in the standard basis of $\mathbb{R}^{n}$.

Let $K$ be the natural cell structure of unit hypercube $[0,1]^{n}$ in $\mathbb{R}^{n}$ and let $K^{d}$ denote its $d$-skeleton. We define the open d-skeleton $F^{d}$ as $K^{d} \backslash K^{d-1}$. Then,

$$
[0,1]^{n}=\bigcup_{d=0}^{n} K^{d}=\bigcup_{d=0}^{n} F^{d}
$$

where the $\bullet$ symbol denotes disjoint union. For example, $[0,1]^{2}$ consists of four points $F^{0}$, four open intervals $F^{1}$ and one open rectangle $F^{2}$.

2.1. Index manipulation. Let $[n]$ denote the ordered set $\{1,2, \ldots, n\}$ that is an index set for the standard basis of $\mathbb{R}^{n}$. We will use ordered sets for indices because the sign of a minor of a matrix is sensitive to the order of indices. Let $A_{I}^{J}$ and $(A)_{I}^{J}$ denote a minor and a submatrix with indices $I$ and $J$ of a matrix $A=\left(a_{i, j}\right)$ respectively.

For example, let $I=\{1,3\}$ and $J=\{2,4\}$. Then

$$
(A)_{I}^{J}=\left[\begin{array}{ll}
a_{1,2} & a_{1,4} \\
a_{3,2} & a_{3,4}
\end{array}\right] \quad \text { and } \quad A_{I}^{J}=\left|\begin{array}{cc}
a_{1,2} & a_{1,4} \\
a_{3,2} & a_{3,4}
\end{array}\right|=\operatorname{det}\left[\begin{array}{cc}
a_{1,2} & a_{1,4} \\
a_{3,2} & a_{3,4}
\end{array}\right] .
$$

Let an ordered set $I=\left\{i_{1}, i_{2}, \ldots, i_{s}\right\} \subset[n]$. Elementary arithmetic operations with an ordered set and a number are done entrywise, for example $2 I-1=\left\{2 i_{1}-1, \ldots, 2 i_{s}-1\right\}$. We call an index $I$ well-ordered if $i_{1}<i_{2}<\cdots<i_{s}$. We consider two different notions of union operation for ordered sets. One is the ordered union $\cup$ respecting the order between two well-ordered indices, for instance, for $t \notin I$,

$$
I \cup\{t\}:=\left\{i_{1}, i_{2}, \ldots, t, \ldots, i_{s}\right\} \quad \text { when } i_{1}<i_{2}<\cdots<t<\cdots<i_{s} .
$$


The other is the joining union $\vee$ as concatenation as follows,

$$
I \vee\{t\}:=\left\{i_{1}, i_{2}, \ldots, i_{s}, t\right\} .
$$

We remark that the joining union is defined no matter whether the constituent sets are well-ordered or not, but the ordered union is defined only for well-ordered sets. In general, the result of a joining union is not well-ordered and might be an ordered multi-set.

We abbreviate a set of one element $\{x\}$ to $x$ omitting the brace symbols, for example, $I \vee\{t\}=: I \vee t$. Let $I$ and $J$ be two ordered sets consisting of the same elements. Then $\sigma(I, J)$ denotes the parity of the permutation between the two ordered sets $I$ and $J$, for example $\sigma(a \vee b, b \vee a)=-1$.

Let $|\cdot|$ and $\|\cdot\|$ denote the cardinality and the total sum of elements of a given set respectively. We remark that $\|\varnothing\|^{k}=0^{k}=1$ when $k=0$. For $\mathbf{v}=\left(v_{1}, v_{2}, \ldots, v_{n}\right)$ in $\mathbb{R}^{n}$, we define the notation $0_{\mathbf{v}}, 1_{\mathbf{v}}, \bullet_{\mathbf{v}}$ and $*_{\mathbf{v}}$ which denote ordered sets of indices satisfying the following:

$$
\begin{array}{ll}
0_{\mathbf{v}}:=\left\{i \in[n] \mid v_{i}=0\right\}, & 1_{\mathbf{v}}:=\left\{i \in[n] \mid v_{i}=1\right\}, \\
*_{\mathbf{v}}:=\left\{i \in[n] \mid v_{i} \neq 0,1\right\}, & \bullet_{\mathbf{v}}:=0_{\mathbf{v}} \cup 1_{\mathbf{v}}=[n] \backslash *_{\mathbf{v}} .
\end{array}
$$

In particular, we define functions $*_{i}: \mathbb{R}^{n} \rightarrow[n]$ and $\bullet_{i}: \mathbb{R}^{n} \rightarrow[n]$ by indicating the $i$-th entry of $*_{\mathbf{v}}$ and $\bullet_{\mathbf{v}}$ of increasing order respectively, i.e.,

$$
\begin{aligned}
& *_{\mathbf{v}}=\left\{i \in[n] \mid v_{i} \neq 0,1\right\}=\left\{*_{1}(\mathbf{v}), *_{2}(\mathbf{v}), \ldots, *_{\left|*_{\mathbf{v}}\right|}(\mathbf{v})\right\} \\
& \bullet_{\mathbf{v}}=\left\{i \in[n] \mid v_{i}=0,1\right\}=\left\{\bullet_{1}(\mathbf{v}), \bullet_{2}(\mathbf{v}), \ldots, \bullet \cdot \bullet_{\mathbf{v}} \mid(\mathbf{v})\right\}
\end{aligned}
$$

When we consider a set of only one element then we omit the index letter like $*(\mathbf{v}):=*_{1}(\mathbf{v})$. To help understanding, let us see an example. Let $\mathbf{v}=\left(0,1, \frac{1}{3}, 0,0, \frac{3}{5}, 1, \frac{1}{8}\right) \in F^{3}$, then we get

$$
\begin{aligned}
& *_{\mathbf{v}}=\{3,6,8\}, \quad 0_{\mathbf{v}}=\{1,4,5\}, \quad 1_{\mathbf{v}}=\{2,7\}, \\
& \left|*_{\mathbf{v}}\right|=3, \quad\left|0_{\mathbf{v}}\right|=3, \quad\left|1_{\mathbf{v}}\right|=2, \\
& \left\|*_{\mathbf{v}}\right\|=3+6+8=17, \quad\left\|0_{\mathbf{v}}\right\|=1+4+5=10, \quad\left\|1_{\mathbf{v}}\right\|=2+7=9, \\
& *_{1}(\mathbf{v})=3, \quad *_{2}(\mathbf{v})=6, \quad *_{3}(\mathbf{v})=8, \\
& \bullet_{1}(\mathbf{v})=1, \quad \bullet_{2}(\mathbf{v})=2, \quad \bullet_{3}(\mathbf{v})=4, \quad \bullet_{3}(\mathbf{v})=5, \quad \bullet_{3}(\mathbf{v})=7 .
\end{aligned}
$$

Finally, we remark that the following always holds by definition:

$$
0_{\mathbf{v}} \cup 1_{\mathbf{v}} \cup *_{\mathbf{v}}=\bullet_{\mathbf{v}} \cup *_{\mathbf{v}}=[n] .
$$

2.2. Hyperplane matrices. Throughout the article, hyperplanes and half spaces are given by

$$
H_{i}:=\left\{\mathbf{x} \mid g_{i}(\mathbf{x})=0\right\} \text { and } H_{i}^{+}:=\left\{\mathbf{x} \mid g_{i}(\mathbf{x}) \geq 0\right\} \text {, }
$$

where the linear coefficients are the following:

$$
\begin{gathered}
g_{1}(\mathbf{x}):=\mathbf{a}_{1} \cdot \mathbf{x}+r_{1}=a_{11} x_{1}+a_{21} x_{2}+\cdots+a_{n 1} x_{n}+r_{1} \\
\vdots \\
g_{m-1}(\mathbf{x}):=\mathbf{a}_{m-1} \cdot \mathbf{x}+r_{m-1}=a_{1, m-1} x_{1}+a_{2, m-1} x_{2}+\cdots+a_{n, m-1} x_{n}+r_{m-1} \\
g_{m}(\mathbf{x}):=\mathbf{a}_{m} \cdot \mathbf{x}+r_{m}=a_{1, m} x_{1}+a_{2, m} x_{2}+\cdots+a_{n, m} x_{n}+r_{m}
\end{gathered}
$$


These coefficients form an $n \times m$ matrix $A$ as the following:

$$
A:=\left(\mathbf{a}_{1}, \mathbf{a}_{2}, \ldots, \mathbf{a}_{m-1}, \mathbf{a}_{m}\right)=\left[\begin{array}{ccccc}
a_{1,1} & a_{1,2} & \cdots & a_{1, k-1} & a_{1, m} \\
a_{2,1} & a_{2,2} & \cdots & a_{2, k-1} & a_{2, m} \\
\vdots & \vdots & & \vdots & \vdots \\
a_{n, 1} & a_{n, 2} & \cdots & a_{n, k-1} & a_{n, m}
\end{array}\right]
$$

In particular, we will take the last $g_{m}(\mathbf{x})$ and $H_{m}$ as the auxiliary function and the auxiliary hyperplane respectively. Let $H^{+}$denote the intersection of all the half spaces $H_{i}^{+}$,

$$
H^{+}=\bigcap_{i \in[m]} H_{i}^{+}
$$

Let $I$ be a set of indices for several hyperplanes usually not including the auxiliary plane, i.e., $I \subset[m-1]$ and let $H_{I}$ denote the intersection of $H^{+} \backslash H_{m}$ and the hyperplanes $H_{i}$ for $i \in I$, i.e.,

$$
H_{I}:=\bigcap_{i \in I} H_{i} \cap H^{+} \backslash H_{m}
$$

We remark that we remove the auxiliary plane $H_{m}$ from the definition of $H_{I}$ because we are going to ignore so-called degenerate vertices (see Section 3.3).

\section{A volume formula for convex polytopes.}

3.1. A review on volume computations. Let us briefly review conceptual methods to compute the exact volume of convex polytopes in a pictorial way. It is well known that the volume of an $n$-parallelotope and an $n$-simplex given by $\mathbf{v}_{1}, \mathbf{v}_{2}, \ldots, \mathbf{v}_{n}$ in $\mathbb{R}^{n}$ are $\left|\operatorname{det}\left(\mathbf{v}_{1} \mathbf{v}_{2} \cdots \mathbf{v}_{n}\right)\right|$ and $\frac{1}{n !}\left|\operatorname{det}\left(\mathbf{v}_{1} \mathbf{v}_{2} \cdots \mathbf{v}_{n}\right)\right|$ respectively. An elementary strategy for computing the volume of a polytope is to decompose the polytope into a signed summation of simplices. In fact, many volume computing algorithms rely entirely on the method of decomposition as in Figure 1. Case (a) is the most obvious decomposition that always exists because of convexity.

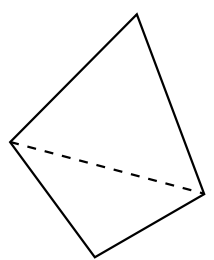

(a)

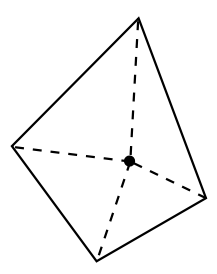

(b)

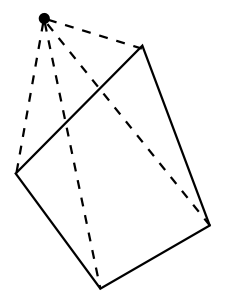

(c)

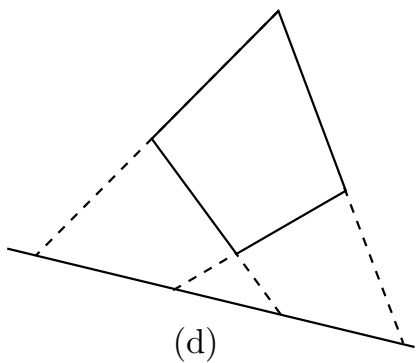

(d)

Figure 1. Typical decomposition methods for a convex polytope.

Cases (b) and (c) are essentially the same except for the position of an auxiliary point. These decompositions are quite elementary but interesting because they make an identity between the volume of a polytope and the volumes of its facets (for example, see J.B. Lasserre [8]). Case (d) is, in some sense, a dual approach to (c) since it uses an auxiliary plane instead of an auxiliary point. 
A volume formula using decomposition (d) was first proposed by J. Lawrence [9] and is a base camp for this study. Let us review the results. A convex compact polytope $P$ in Euclidean $n$-space is given by

$$
P=\bigcap_{i \in[m]} H_{i}^{+} \subset \mathbb{R}^{n}
$$

Consider an auxiliary function $f(\mathbf{x})$ with a hyperplane $f(\mathbf{x})=0$. Then we need two requirements as follows.

\section{LAWRENCE'S TWO CONDITIONS.}

simplicity All vertices of a polytope $P$ are simple, which means that the degrees of vertices in $P$ are the same as the dimension of $P$.

non-parallelness The auxiliary function $f$ is non-constant on each edge of $P$.

Now, we have the following volume formula.

Theorem 3.1. (J. Lawrence) If P satisfies Lawrence's condition, the volume is computed by

$$
\operatorname{vol}(P)=\sum_{\mathbf{v}: \text { a vertex of } P} N_{\mathbf{v}} \quad \text { with } \quad N_{\mathbf{v}}=\frac{f(\mathbf{v})^{n}}{n ! \delta_{\mathbf{v}} \gamma_{1} \gamma_{2} \cdots \gamma_{n}},
$$

where $\delta_{\mathbf{v}}$ is the absolute value of the determinant of the $n \times n$ matrix whose columns are $-\mathbf{a}_{i_{1}},-\mathbf{a}_{i_{2}}, \ldots,-\mathbf{a}_{i_{n}}$. Here, a vertex $\mathbf{v}$ of $P$ is the intersection of $H_{i_{1}}, \ldots, H_{i_{n}}$ with $i_{1}<i_{2}<\cdots<i_{n}$ and $\gamma_{1}, \gamma_{2}, \ldots, \gamma_{n}$ are obtained by $\mathbf{a}_{m}=-\left(\gamma_{1} \mathbf{a}_{i_{1}}+\cdots+\gamma_{n} \mathbf{a}_{i_{n}}\right)$.

For a more detailed description, see J. Lawrence [9]. From a geometric point of view, each $N_{\mathbf{v}}$ corresponds to a signed volume of a simplex which is projected from a vertex $\mathbf{v}$ to the plane $\left\{\mathbf{x} \in \mathbb{R}^{n} \mid f(\mathbf{x})=0\right\}$ as in Case (d) in figure 1. Note that the projected simplex possibly has infinite volume, but non-parallelness guarantees that all vertices of the simplex are located in a bounded region of $\mathbb{R}^{n}$, not at infinity)

Note that the non-parallel condition is a sufficient condition but not a necessary condition for the volume of a projected simplex not to be $\infty$. By observation we found a case that violates the requirements, where we can exploit Lawrence's formula without an extra effort. Before doing that, we review his formula concretely in the following section.

3.2. An explicit form of Lawrence's formula. Lawrence's formula has sometimes been expressed in a quite explicit form in the literature (for example, see [6, p.393]). But the known formulations are still not enough to proceed for our purpose. So we rewrite his formula in a closed form explicitly with linear coefficients. From now on, we put an auxiliary plane as the last hyperplane $H_{m}$. In this section, $H_{m}$ is redundant, i.e., $P=P \cap H_{m}^{+}$and $H_{m} \neq H_{i}$ for any $i \in[m-1]$. We remark that $m-1>n$ holds in order to make a convex compact polytope.

TheOrem 3.2. Let a convex polyhedron $P=\bigcap_{i \in[m-1]} H_{i}^{+}$satisfy Lawrence's conditions. Then the volume is

$$
\operatorname{vol}(P)=\sum_{\substack{I \subset[m-1] \\|I|=n}} \sum_{\mathbf{v} \in H_{I}} \frac{(-1)^{\frac{n(n+1)}{2}}\left(g_{m}(\mathbf{v}) A_{[n]}^{I}\right)^{n}}{n !\left|A_{[n]}^{I}\right| \prod_{t \in I} A_{[n]}^{I \backslash t \cup m}}
$$


REMARK 3.3. In the formula, the second summation consists of either an empty summand or only one summand. In spite of the redundancy of the expression, we persist in this inefficient form to be compatible with the main theorem on a clipped hypercube. We remark that $I \backslash t \cup m=I \backslash t \vee m$ because $m$ is the last element.

Proof of Theorem 3.2. Let us just do a direct computation in our setup from Lawrence's formula (3.3). Each vertex $\mathbf{v}$ is an intersection of exactly $n$ hyperplanes other than the auxiliary plane,

$$
H_{i_{1}}, H_{i_{2}}, \ldots, H_{i_{n}} \quad\left(1 \leq i_{1}<i_{2}<\cdots<i_{n} \leq m-1\right)
$$

with $\mathbf{v}=\bigcap_{t=1}^{n} H_{i_{t}}$. Let $I:=I_{\mathbf{v}}:=\left(i_{1}, \ldots, i_{n}\right)$. Then

$$
(-A)_{[n]}^{I}=\left(-\mathbf{a}_{i_{1}},-\mathbf{a}_{i_{2}}, \ldots,-\mathbf{a}_{i_{n}}\right)
$$

Let $\gamma:=\gamma_{\mathbf{v}}:=\left(\gamma_{1}, \ldots, \gamma_{n}\right)^{t}$. It follows from the definition in [9] that $\gamma$ is defined as satisfying

$$
\mathbf{a}_{m}=(-A)_{[n]}^{I} \gamma
$$

So, we have $\gamma=\left((-A)_{[n]}^{I}\right)^{-1} \mathbf{a}_{m}$.

Let $\left(x_{\mu \nu}\right):=\left((-A)_{[n]}^{I}\right)^{-1}$. Then by Cramer's rule we get $x_{\mu \nu}=\frac{(-1)^{\mu+\nu}\left((-A)_{[n]}^{I}\right)_{\nu, \mu}}{\operatorname{det}\left((-A)^{I}\right)}$, where $\left((-A)_{[n]}^{I}\right)_{\nu, \mu}$ means the $(\nu, \mu)$-minor of the submatrix $(-A)_{[n]}^{I}$, i.e., $\left|(-A)_{[n] \backslash \nu}^{I \backslash i_{\mu}}\right|$ with $I=\left\{i_{1}, i_{2}, \ldots, i_{n}\right\}$. Then

$$
\begin{gathered}
\gamma=\left[\begin{array}{c}
\sum_{j} a_{j, m} x_{1, j} \\
\sum_{j} a_{j, m} x_{2, j} \\
\vdots \\
\sum_{j} a_{j, m} x_{n, j}
\end{array}\right]=\frac{1}{\operatorname{det}\left((-A)^{I}\right)}\left[\begin{array}{c}
\sum_{j}(-1)^{1+j} a_{j, m}\left((-A)_{[n]}^{I}\right)_{j, 1} \\
\sum_{j}(-1)^{2+j} a_{j, m}\left((-A)_{[n]}^{I}\right)_{j, 2} \\
\vdots \\
\sum_{j}(-1)^{n+j} a_{j, m}\left((-A)_{[n]}^{I}\right)_{j, n}
\end{array}\right] \\
=\frac{1}{\operatorname{det}\left((-A)^{I}\right)}\left[\begin{array}{c}
\operatorname{det}\left(\mathbf{a}_{m},-\mathbf{a}_{i_{2}}, \ldots,-\mathbf{a}_{i_{n}}\right) \\
\operatorname{det}\left(-\mathbf{a}_{i_{1}}, \mathbf{a}_{m}, \ldots,-\mathbf{a}_{i_{n}}\right) \\
\vdots \\
\operatorname{det}\left(-\mathbf{a}_{i_{1}},-\mathbf{a}_{i_{2}}, \ldots, \mathbf{a}_{m}\right)
\end{array}\right]
\end{gathered}
$$

Hence,

$$
\begin{aligned}
\gamma_{1} \gamma_{2} \cdots \gamma_{n} & =\prod_{j=1}^{n} \frac{\operatorname{det}\left(-\mathbf{a}_{i_{1}}, \ldots,-\mathbf{a}_{i_{j-1}}, \mathbf{a}_{m},-\mathbf{a}_{i_{j+1}}, \ldots,-\mathbf{a}_{i_{n}}\right)}{\operatorname{det}\left((-A)_{[n]}^{I}\right)} \\
& =\prod_{j=1}^{n} \frac{(-1)^{n-1} \operatorname{det}\left(\mathbf{a}_{i_{1}}, \ldots, \mathbf{a}_{i_{j-1}}, \mathbf{a}_{m}, \mathbf{a}_{i_{j+1}}, \ldots, \mathbf{a}_{i_{n}}\right)}{(-1)^{n} \operatorname{det}\left(A_{[n]}^{I}\right)} \\
& =(-1)^{n}(-1)^{\frac{n(n-1)}{2}} \prod_{j=1}^{n} \frac{\operatorname{det}\left(\mathbf{a}_{i_{1}}, \ldots, \mathbf{a}_{i_{j-1}}, \mathbf{a}_{i_{j+1}}, \ldots, \mathbf{a}_{i_{n}}, \mathbf{a}_{m}\right)}{\operatorname{det}\left(A_{[n]}^{I}\right)} \\
& =\frac{(-1)^{\frac{n(n+1)}{2}}}{\operatorname{det}\left(A_{[n]}^{I}\right)^{n}} \prod_{t \in I} A_{[n]}^{I \backslash t \cup m} .
\end{aligned}
$$

Also we get

$$
\delta_{\mathbf{v}}=\left|\operatorname{det}\left((-A)_{[n]}^{I}\right)\right|=\left|\operatorname{det}\left(A_{[n]}^{I}\right)\right|=\left|A_{[n]}^{I}\right| .
$$


3.3. Degenerate vertices and edges. Let us consider the situation of Theorem 3.2 such that the last hyperplane $H_{m}$ is not redundant. In other words we choose an auxiliary plane $\{f(\mathbf{x})=0\}$ as the hyperplane $H_{m}=\left\{\mathbf{x} \mid g_{m}(\mathbf{x})=0\right\}$, when a polytope $P$ is given by a non-redundant intersection of $H_{i}$ 's for $i \in[m]$, i.e., $P \neq \bigcap_{i \in[m-1]} H_{i}^{+}$. Then let us look at the following brief formula,

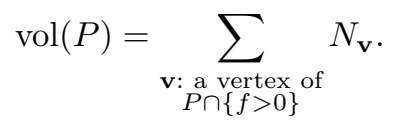

The formula looks almost the same as Lawrence's original one, but ignores any vertex placed on $H_{m}$. Recall the geometric interpretation that $N_{\mathbf{v}}$ is the volume of a projected simplex. Intuitively, $N_{\mathbf{v}}$ for $f(\mathbf{v})=0$ seems to vanish. If this is true, we should be able to handle some situations violating Lawrence's condition, i.e., if a non-simple vertex or a parallel edge in $P$ is placed on an auxiliary plane, it is enough to simply ignore $N_{\mathbf{v}}$ as in (3.4). We introduce a term to describe the situation as follows.

Definition 3.4. A vertex or an edge of $P$ is degenerate into a hyperplane $H_{m}$ if the vertex or the edge belongs to $H_{m}$, or otherwise non-degenerate. We usually omit indicating $H_{m}$ unless it will cause confusion.

Now we verify the validity of the above intuition in the following theorem.

ThEOREM 3.5. Let a convex polyhedron $P=\bigcap_{i \in[m-1]} H_{i}^{+} \cap H_{m}^{+}$where Lawrence's conditions hold only for non-degenerate vertices and edges. Then the volume is given by

$$
\operatorname{vol}(P)=\sum_{\substack{I \subset[m-1] \\|I|=n}} \sum_{\mathbf{v} \in H_{I}} \frac{(-1)^{\frac{n(n+1)}{2}}\left(g_{m}(\mathbf{v}) A_{[n]}^{I}\right)^{n}}{n !\left|A_{[n]}^{I}\right| \prod_{t \in I} A_{[n]}^{I \backslash t \cup m}} .
$$

REMARK 3.6. Note that the formula itself is exactly the same as Theorem 3.2 except the term $N_{\mathbf{v}}$ for any non-degenerate $\mathbf{v}$ isn't contained in the summation. The number of hyperplanes involved in the computation is also reduced by one. In particular, the two requirements are practically weakened, i.e., it is enough to check whether simplicity and non-parallelness are fulfilled only at non-degenerate vertices and edges.

Proof of Theorem 3.5. The proof consists of two steps. We first prove it for the case violating only non-parallelness, i.e., we assume that $P$ has only simple vertices. Consider a vector $\mathbf{q}$ that is not parallel to any edge of $P$ and choose an auxiliary function $f_{\epsilon}$ as follows,

$$
f_{\epsilon}(\mathbf{x}):=\left(\mathbf{a}_{m}+\epsilon \mathbf{q}\right) \cdot \mathbf{x}+r_{m}
$$

Then we can apply Theorem 3.2 to obtain $\operatorname{vol}(P)$ by putting

$$
H_{m+1}:=\left\{\mathbf{x} \mid f_{\epsilon}(\mathbf{x})=0\right\}
$$

as an auxiliary plane. Let the volume of $P$ and the value of Theorem 3.2 be $\operatorname{denoted}_{\text {by }} \operatorname{vol}(P)$ and $\operatorname{vol}_{\epsilon}(P)$ respectively. By Lawrence, we have $\operatorname{vol}(P)=\operatorname{vol}_{\epsilon}(P)$ for any $\epsilon>0$ and the $\operatorname{vol}_{\epsilon}(P)$ is exactly represented by

$$
\operatorname{vol}_{\epsilon}(P)=\sum_{\substack{I \subset[m] \\|I|=n}} \sum_{\mathbf{v} \in H_{I}} \frac{(-1)^{\frac{n(n+1)}{2}}\left(g_{m+1}(\mathbf{v}) A_{[n]}^{I}\right)^{n}}{n !\left|A_{[n]}^{I}\right| \prod_{t \in I} A_{[n]}^{I \backslash t \cup(m+1)}}
$$


One needs to be careful here: The number of hyperplanes increases by one from the count of Theorem 3.2 , i.e., $m \mapsto m+1$. We decompose the summation into two parts according to whether $\mathbf{v}$ belongs to $H_{m}$ or not.

$$
\begin{aligned}
= & \sum_{\substack{I \subset[m-1] \\
|I|=n}} \sum_{\mathbf{v} \in H_{I}} \frac{(-1)^{\frac{n(n+1)}{2}}\left(g_{m+1}(\mathbf{v}) A_{[n]}^{I}\right)^{n}}{n !\left|A_{[n]}^{I}\right| \prod_{t \in I} A_{[n]}^{I \backslash t \cup(m+1)}} \\
& +\sum_{\substack{\mathbf{v} \in H_{I} \cup m \\
|I|=n-1}} \frac{(-1)^{\frac{n(n+1)}{2}}\left(g_{m+1}(\mathbf{v}) A_{[n]}^{I \cup m}\right)^{n}}{n !\left|A_{[n]}^{I \cup m}\right| \prod_{t \in I \cup m} A_{[n]}^{I \cup m \backslash t \cup(m+1)}} .
\end{aligned}
$$

Let us look at the first part (3.7). The summand $N_{\mathbf{v}}^{\epsilon}$ for each vertex $\mathbf{v} \in H_{I} \backslash H_{m}$ goes to $N_{\mathbf{v}}$ in (3.5), i.e.,

$$
\begin{aligned}
\lim _{\epsilon \rightarrow 0} N_{\mathbf{v}}^{\epsilon} & =\lim _{\epsilon \rightarrow 0} \sum_{\substack{I \subset[m-1] \\
|I|=n}} \sum_{\mathbf{v} \in H_{I}} \frac{(-1)^{\frac{n(n+1)}{2}}\left(g_{m+1}(\mathbf{v}) A_{[n]}^{I}\right)^{n}}{n !\left|A_{[n]}^{I}\right| \prod_{t \in I} A_{[n]}^{I \backslash t \cup(m+1)}} \\
& =\sum_{\substack{I \subset[m-1] \\
|I|=n}} \sum_{\mathbf{v} \in H_{I}} \frac{(-1)^{\frac{n(n+1)}{2}}\left(g_{m}(\mathbf{v}) A_{[n]}^{I}\right)^{n}}{n !\left|A_{[n]}^{I}\right| \prod_{t \in I} A_{[n]}^{I \backslash t \cup m}}=N_{\mathbf{v}} .
\end{aligned}
$$

Therefore, the first part (3.7) goes exactly to the formula of (3.5) as $\epsilon \rightarrow 0$.

Now we show that the second part (3.8) goes to zero as $\epsilon \rightarrow 0$. For $\mathbf{v} \in H_{m}$, we have $g_{m+1}(\mathbf{v})=f_{\epsilon}(\mathbf{v})=$ $\epsilon \mathbf{q} \cdot \mathbf{v}$ and

$$
A_{[n]}^{I \cup m \backslash t \cup(m+1)}=\operatorname{det}\left(\mathbf{a}_{i_{1}}, \ldots, \mathbf{a}_{i_{t-1}}, \mathbf{a}_{i_{t+1}}, \ldots, \mathbf{a}_{m}, \mathbf{a}_{m}+\epsilon \mathbf{q}\right)
$$

From this, we obtain

$$
\begin{aligned}
\prod_{t \in I \cup m} A_{[n]}^{I \cup m \backslash t \cup(m+1)}= & \epsilon^{n-1} A_{[n]}^{I \cup m} \prod_{t=1}^{n-1} \operatorname{det}\left(\mathbf{a}_{i_{1}}, \ldots, \mathbf{a}_{i_{t-1}}, \mathbf{a}_{i_{t+1}}, \ldots, \mathbf{a}_{m}, \mathbf{q}\right) \\
& +\epsilon^{n} \operatorname{det}\left(\mathbf{a}_{i_{1}}, \ldots, \mathbf{a}_{i_{n-1}}, \mathbf{q}\right) \prod_{t=1}^{n-1} \operatorname{det}\left(\mathbf{a}_{i_{1}}, \ldots, \mathbf{a}_{i_{t-1}}, \mathbf{a}_{i_{t+1}}, \ldots, \mathbf{a}_{m}, \mathbf{q}\right)
\end{aligned}
$$

Using these computations, we know that the denominator and the numerator of $N_{\mathbf{v}}^{\epsilon}$ in the expression (3.8) have dominating terms of $\epsilon^{n-1}$ and $\epsilon^{n}$, respectively. Therefore, we conclude that the second part goes to zero as $\epsilon \rightarrow 0$.

Now we can freely use the formula (3.5) for a simple polytope $P$ even cases violating the non-parallel condition as long as the edge is degenerate into $H_{m}$. The remaining case is that $P$ has a non-simple vertex which is degenerate into $H_{m}$. Let us consider a truncated polytope $P^{\epsilon}$ obtained from $P$ by cutting out a sufficiently small neighborhood of each vertex $\mathbf{v}$ by an additional hyperplane $H_{\mathbf{v}}^{\epsilon}$. More precisely, a nonsimple vertex $\mathbf{v}$ is removed from $P$ and a new facet $H_{\mathbf{v}}^{\epsilon}$ appears with several simple vertices w's such that (i) the normal vector of $H_{\mathbf{v}}^{\epsilon}$ is fixed as $\epsilon \rightarrow 0$, (ii) when $\epsilon=0, H_{\mathbf{v}}^{\epsilon}$ contains $\mathbf{v}$, and (iii) $f(\mathbf{w})<\epsilon$ for any new vertex $\mathbf{w}$ from the truncation by $H_{\mathbf{v}}^{\epsilon}$. 
Let $\operatorname{vol}^{f}(P)$ be the value of the formula (3.5). We estimate vol ${ }^{f}(P)$ as follows,

$$
\operatorname{vol}(P)-\operatorname{vol}^{f}(P)=\operatorname{vol}(P)-\operatorname{vol}^{f}\left(P^{\epsilon}\right)+\sum_{\mathbf{w}} N_{\mathbf{w}}^{\epsilon}
$$

where $N_{\mathbf{w}}^{\epsilon}$ is the summand of $\operatorname{vol}^{f}\left(P^{\epsilon}\right)$ at $\mathbf{w}$ of $(3.5)$. Note that we know that vol ${ }^{f}\left(P^{\epsilon}\right)=\operatorname{vol}\left(P^{\epsilon}\right) \operatorname{since} P^{\epsilon}$ fulfills the simplicity condition. So the difference $\operatorname{vol}(P)-\operatorname{vol}^{f}\left(P^{\epsilon}\right)$ becomes $\operatorname{vol}(P)-\operatorname{vol}\left(P^{\epsilon}\right)$ which obviously goes to zero as $\epsilon \rightarrow 0$ since they are geometric Euclidean volumes. Moreover, $N_{\mathbf{w}}^{\epsilon}$ also goes to zero since $N_{\mathbf{w}}^{\epsilon}=c \epsilon^{n}$ for some constant $c$ because the normal vector of $H_{\mathbf{v}}^{\epsilon}$ is fixed while $\epsilon \rightarrow 0$. Therefore, we conclude that even if $P$ contains a non-simple vertex, $\operatorname{vol}(P)=\operatorname{vol}^{f}(P)$ because of the degeneracy of the vertex $\mathbf{v}$.

3.4. $\epsilon$-pertubation for general polytopes. Sometimes we want to consider the volume of a general polytope that might violate Lawrence's conditions. For example, a way to apply Lawrence's formula to a non-simple polytope is to decompose a non-simple vertex into projected simplices along "lexicographic rule" $([6],[9])$.

In the previous section, our formula weakened Lawrence's conditions in a specific situation. However, the strategy using a limiting process can be, in principle, utilized in general. Here we discuss such a ubiquitous method, called $\epsilon$-perturbation, for cases violating either simplicity or non-parallelness. Let us say a polytope is good if it satisfies Lawrence's conditions, and otherwise bad.

The following two facts constitute the essential justification for the $\epsilon$-perturbation method.

1. The volume of a clipped hypercube is a continuous function on the parameter spaces of the hyperplanes.

2. Lawrence's condition is an open condition. Precisely, the set of bad polytopes is a Zariski-closed subset in the parameter space.

Therefore, we can always find a good polytope which has only an arbitrarily small difference from the original bad polytope. Moreover, the continuity of volume tells us that the volume difference is also arbitrarily small.

Let us explain the strategy more precisely as follows. For a bad polytope $P$, let us add a perturbation variable $\epsilon$ (or sometimes more hyerperplanes) into a system of hyperplanes such that the perturbed polytopes $P_{\epsilon}$ are always good for any sufficiently small $\epsilon>0$. Then we can obtain the volume of $P$ as the limit as $\epsilon \rightarrow 0$ by only using the formula for good polytopes.

REMARK 3.7. In fact, the strategy itself is nothing special but it is usually not easy because the analysis of a precise limit is difficult in many cases. In particular, to apply this, a sufficiently concrete expression is required as in Theorem 3.5 and Section 5. We are going to give more examples of $\epsilon$-perturbation in Section 6 and the Appendix. In a follow-up article [3], a concrete exposition of $\epsilon$-perturbation for several cases are investigated.

4. Volume formulas for a clipped hypercube. Now we derive volume formulas for a clipped hypercube. A clipped hypercube has two types of hyperplanes: the facets of $[0,1]^{n}$ and the clipping hyperplanes $H_{1}, \ldots, H_{m}$. We use calligraphic notation $\mathcal{H}, \mathcal{A}$ and $\mathcal{I}$ when we consider both types of hyperplanes in a unified way. 
4.1. Hyperplanes and indices for clipped hypercubes. An $n$-dimensional unit hypercube $[0,1]^{n}$ is given by $2 n$ half spaces,

$$
x_{i} \geq 0 \quad \text { and } \quad x_{i} \leq 1 \quad \text { for } i \in[n]
$$

Let $P$ be a hypercube clipped by hyperplanes $H_{1}, \ldots H_{m}$, i.e.,

$$
P:=[0,1]^{n} \cap \bigcap_{i=1}^{m} H_{i}^{+}
$$

From now on, we use separate notation $\mathcal{H}_{i}$ and $H_{i}$ for hyperplanes. The former $\mathcal{H}_{i}$ indicates a hyperplane in the set of all hyperplanes of $P$, while the later $H_{i}$ indicates a hyperplane in the subset of only the clipping hyperplanes. We follow the same notational conventions for $\mathcal{H}, \mathcal{H}^{+}$and $\mathcal{H}_{i}$ and so on, as in Section 2.2. Let us consider the set of half spaces $\mathcal{H}_{i}^{+}$. In order to apply Theorem 3.2, consider

$$
\mathcal{H}_{i}^{+}=\left\{\begin{array}{cl}
\left\{\mathbf{x} \in \mathbb{R}^{n} \mid \mathbf{e}_{(i+1) / 2} \cdot \mathbf{x} \geq 0\right\}, & \text { if } i \in[2 n] \text { is odd, } \\
\left\{\mathbf{x} \in \mathbb{R}^{n} \mid\left(-\mathbf{e}_{i / 2}\right) \cdot \mathbf{x}+1 \geq 0\right\}, & \text { if } i \in[2 n] \text { is even, } \\
H_{i-2 n}^{+}, & \text {if } i \in[2 n+m] \backslash[2 n] .
\end{array}\right.
$$

We also consider a big coefficient matrix $\mathcal{A}$ and the index set $\mathcal{I}=[2 n+m]$ for $\mathcal{H}_{i}$, where the matrix $A$ is given by hyperplanes $H_{i}$ and the index set $I=[m]$. We construct an $n \times(2 n+m)$ matrix $\mathcal{A}$ which is determined by $\mathcal{H}$ as follows. Let us keep in mind the canonical embedding $I$ into $\mathcal{I}$ by $i \mapsto i+2 n$.

$$
\begin{aligned}
& \mathcal{A}=\left(\mathbf{e}_{1},-\mathbf{e}_{1}, \mathbf{e}_{2},-\mathbf{e}_{2}, \ldots, \mathbf{e}_{n},-\mathbf{e}_{n} \mid A\right)
\end{aligned}
$$

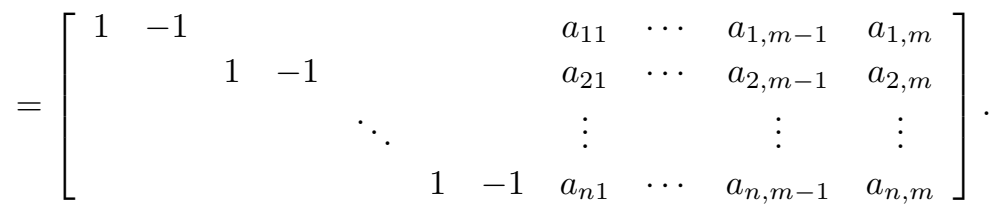

For a simple vertex $\mathbf{v}$, there is an index set $\mathcal{I}$ tracking the $n$ hyperplanes $\mathcal{H}_{i_{1}}, \mathcal{H}_{i_{2}}, \ldots, \mathcal{H}_{i_{n}}$ making up the vertex v, i.e.,

$$
\mathbf{v}=\bigcap_{k \in \mathcal{I}} \mathcal{H}_{k} \quad \text { for } \mathcal{I}=\mathcal{I}(\mathbf{v})=\left\{i_{1}, \ldots, i_{n}\right\} \subset[2 n+m]
$$

where $\mathcal{I}$ is well-ordered, $i_{1}<i_{2}<\cdots<i_{n}$. We decompose $\mathcal{I}$ into two parts,

$$
\mathcal{I}=\mathcal{I}_{01} \bullet \mathcal{I}_{*}
$$

with $\mathcal{I}_{01}=\{i \in \mathcal{I} \mid i \in[2 n]\}$ and $\mathcal{I}_{*}=\{i \in \mathcal{I} \mid i \in[2 n+m] \backslash[2 n]\}$. We can check that

$$
(\mathcal{A})_{[n]}^{\mathcal{I}_{*}}=(A)_{[n]}^{I} .
$$

The following lemma says that each vertex has a natural grading from $F^{d}$. Our volume formula is a summation over the grading.

LEMMA 4.1. For a simple vertex $\mathbf{v}$ of a clipped hypercube $P$, there is an index set $I \subset[n]$ such that $\mathbf{v} \in F^{d} \cap H_{I}$. Moreover, $\left|*_{\mathbf{v}}\right|=|I|=d$. 
Proof. Let $\mathbf{v}=\left(v_{1}, v_{2}, \ldots, v_{n}\right) \in F^{d}$, then $\left|\bullet_{\mathbf{v}}\right|=\left|\mathcal{I}_{01}\right|=n-d$ because $v_{i}=0$ or 1 if and only if $\mathbf{v}$ intersects a hyperplane of form $x_{i}=0$ or $x_{i}=1$. Since $\mathcal{I}_{*}=I+2 n$ and $\left|\mathcal{I}_{*}\right|=|I|$, the lemma is obvious by (2.2) and (4.11).

Let $\mathbf{v} \in F^{d}$ and let us consider this in more detail,

$$
\mathbf{v} \in \bigcap_{i \in 0_{\mathbf{v}}} \mathcal{H}_{2 i-1} \cap \bigcap_{i \in 1_{\mathbf{v}}} \mathcal{H}_{2 i}
$$

Let $\mathcal{I}_{0}:=\left\{i \in \mathcal{I}_{01} \mid i\right.$ is odd $\} \quad$ and $\quad \mathcal{I}_{1}:=\left\{i \in \mathcal{I}_{01} \mid i\right.$ is even $\}$. Then it immediately follows that

$$
\mathcal{I}_{0}=\left(2 \cdot 0_{\mathbf{v}}-1\right) \quad \text { and } \quad \mathcal{I}_{1}=2 \cdot 1_{\mathbf{v}} .
$$

Lastly, we also obtain the following lemma.

Lemma 4.2. $(\mathcal{A})_{\bullet_{\mathrm{v}}}^{\mathcal{I}_{01}}$ is a diagonal matrix with

$$
\left((\mathcal{A})_{\bullet_{\mathbf{v}}}^{\mathcal{I}_{01}}\right)_{i, i}=\left\{\begin{aligned}
1, & \text { if } v_{\bullet_{i}(\mathbf{v})}=0, \\
-1, & \text { if } v_{\bullet_{i}(\mathbf{v})}=1 .
\end{aligned}\right.
$$

In particular, $\mathcal{A}_{\bullet_{\mathbf{v}}}^{\mathcal{I}_{01}}=(-1)^{\left|1_{\mathbf{v}}\right|}$.

Proof. The hyperplanes $\mathcal{H}_{2 i}$ and $\mathcal{H}_{2 i-1}$ are parallel and never intersect. Hence, for any $t \in[n], 2 t$ and $2 t-1$ cannot be contained in $\mathcal{I}_{01}$ at the same time. By the definition of $\mathcal{A}$ and (4.14), the matrix is diagonal with $\left|0_{\mathbf{v}}\right|$ entries equal to 1 and $\left|1_{\mathbf{v}}\right|$ entries equal to -1 .

4.2. Good clipping conditions. We consider two more explicit conditions called good clipping conditions in which a volume formula is applicable. They look almost identical to Lawrence's two conditions in a concrete form, but are not exactly the same. First of all, we don't need any requirement for a degenerate vertex or edge since Theorem 3.5 doesn't require them. So a polytope violating simplicity or non-parallelness may satisfy our good clipping conditions. Not only that, but Lawrence's condition doesn't imply the good clipping conditions. To see this concretely, see Example 4.4.

Proposition 4.3. (Good clipping conditions) If a clipped hypercube $P$ satisfies the following assumptions:

(A) for any $I \subset[m-1], F^{|I|-1} \cap H_{I}=\varnothing$,

(B) for any $I \subset[m-1]$ and $\mathbf{v} \in F^{|I|} \cap H_{I}, \prod_{t \in I} A_{*_{\mathrm{v}}}^{I \cup m \backslash t} \prod_{t \in \bullet_{\mathrm{v}}} A_{*_{\mathrm{v}} \cup t}^{I \cup m} \neq 0$,

then Lawrence's two conditions hold for non-degenerate vertices.

Proof. Let us see that (A) implies that every non-degenerate vertex is simple. If a non-simple vertex $\mathbf{v}$ exists then there are at least $n+1$ hyperplanes intersecting $\mathbf{v}$, which meet $k$ hyperplanes of $[0,1]^{n}$ and $H_{i_{1}}, \ldots, H_{i_{n-k+1}}$ for $0 \leq k \leq n+1$. Then $\mathbf{v} \in F^{n-k} \cap H_{I}$ with $I=\left\{i_{1}, \ldots, i_{n-k+1}\right\}$. This conflicts with (A). Condition (B) means the volume $N_{\mathbf{v}}$ of the projected simplex at $\mathbf{v}$ is finite, which is equivalent to Lawrence's non-parallelness.

Let us give an example of a polytope $P$ which violates the good clipping condition (A) but satisfies Lawrence's simplicity. Note the non-parallel condition always guarantees good clipping condition (B). 
ExAMPLE 4.4. There is a clipped hypercube which is simple but violates the good clipping condition (A). Consider $P=[0,1]^{3} \cap H_{1}^{+} \cap H_{2}^{+}$where

$$
\begin{aligned}
& H_{1}^{+}:=\left\{\left(x_{1}, x_{2}, x_{3}\right) \in \mathbb{R}^{3} \mid x_{1}+x_{2}+2 x_{3} \geq 1\right\}, \\
& H_{2}^{+}:=\left\{\left(x_{1}, x_{2}, x_{3}\right) \in \mathbb{R}^{3} \mid x_{1}+x_{2} \leq 1\right\} .
\end{aligned}
$$

We can see that all vertices are simple, but

$$
\begin{aligned}
& H_{1} \cap F^{1-1}=\{(1,0,0),(0,1,0)\} \neq \varnothing \\
& H_{2} \cap F^{1-1}=\{(1,0,0),(0,1,0),(1,0,1),(0,1,1)\} \neq \varnothing .
\end{aligned}
$$

In this example, we observe that there can be a simple vertex in a clipped hypercube, which lies in the intersection of more than $n+1$ hyperplanes in $\mathcal{H}$. When we enumerate hyperplanes at a vertex of a clipped hypercube, the hyperplanes in $[0,1]^{n}$ are chosen by a default, but some of them are sometimes unnecessary because of clipping hyperplanes in $H$.

Finally, we remark that Conditions (A) and (B) are also open conditions like Lawrence's conditions.

4.3. Several volume formulas. We now present our main formulas. Refer to Section 2 and 4.1 for notation. We will postpone the proof until later. If one wants to obtain each summand up to sign, the evaluation is much simpler. However, we have to keep track of correct signs to derive an explicit formula. The practical difficulty of the proof comes from handling the complicated signs, and hence, we need to prepare something to deal with the signs properly.

Here, we are going to discuss several different presentations of the same formula and the relevant lemmas to convert among the formulas using different conventions. Let us look at a first expression for the volume of clipped hypercube as follows.

THEOREM 4.5. The volume of a hypercube clipped by $m$ halfspaces $H_{1}, H_{2}, \ldots, H_{m}$ satisfying good clipping conditions is given by

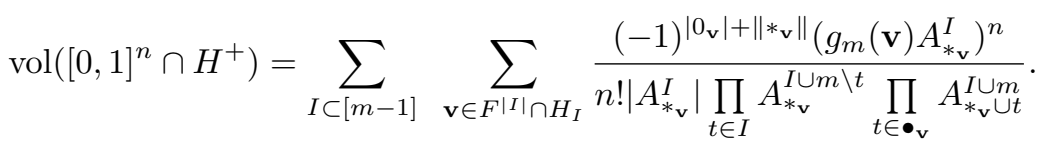

We consider another expression which uses joining union $\vee$ instead of ordered union $\cup$ as follows. The only difference between them is the sign computation for $\frac{|I|(|I|+1)}{2}$ and $\left\|*_{\mathbf{v}}\right\|$.

THEOREM 4.6. The volume of a hypercube clipped by $m$ halfspaces $H_{1}, H_{2}, \ldots, H_{m}$ satisfying good clipping conditions is given by

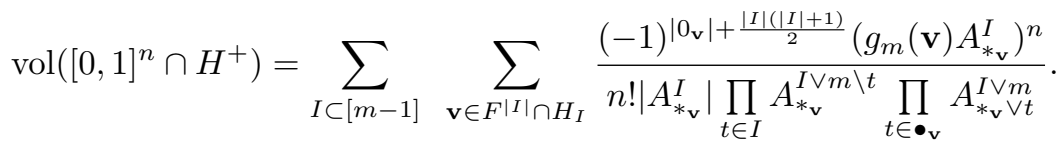

The following lemma implies that the above two theorems are equivalent.

LEMMA 4.7.

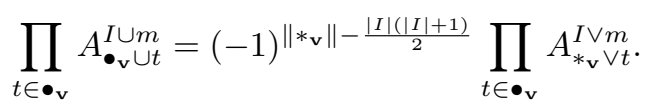


Proof. The set $\bullet_{\mathbf{v}}=[n] \backslash *_{\mathbf{v}}$ is divided into $|I|+1$ (possibly empty) blocks,

$$
\begin{gathered}
\left\{1,2, \ldots, i_{1}-1\right\}, \\
\left\{i_{1}+1, i_{1}+2, \ldots, i_{2}-1\right\}, \\
\vdots \\
\left\{i_{|I|}+1, i_{|I|}+2, \ldots, n\right\} .
\end{gathered}
$$

Whenever $t \in \bullet_{\mathbf{v}}$ is placed in each block, it requires $|I|,|I|-1, \ldots, 1,0$ transpositions. Hence, the total number of transpositions is

$$
\begin{aligned}
& \left(i_{1}-1\right)|I|+\left(i_{2}-i_{1}-1\right)(|I|-1)+\cdots+\left(i_{|I|}-i_{|I|-1}-1\right) 1+\left(n-i_{|I|}\right) 0 \\
& =i_{1}+i_{2} \cdots+i_{|I|}-(|I|+(|I|-1)+\cdots+1) \\
& =\left\|*_{\mathbf{v}}\right\|-\frac{|I|(|I|+1)}{2}
\end{aligned}
$$

Recall that the ordered union is commutative but the joining union is not, i.e., $I \cup m=m \cup I$ but $I \vee m \neq m \vee I$. Thus, in Theorem 4.5 the expression order of the union operations doesn't matter. But if one takes the joining union as in Theorem 4.6, there are several choices of expressions because it is sensitive to changing order. The following lemma shows that orders of the expressions affects a few things in Theorem 4.6 .

LEMMA 4.8.

$$
\begin{aligned}
& \prod_{t \in I} A_{*_{\mathrm{v}}}^{I \vee m \backslash t}=\prod_{t \in I} A_{*_{\mathrm{v}}}^{m \vee \backslash \backslash t}=\prod_{t \in I} A_{*_{\mathrm{v}}}^{I \cup m \backslash t}=\prod_{t \in I} A_{*_{\mathrm{v}}}^{m \cup \backslash \backslash t}, \\
& \prod_{t \in \bullet_{\mathrm{v}}} A_{*_{\mathrm{v}} \vee t}^{I \vee m}=\prod_{t \in \bullet_{\mathrm{v}}} A_{t \vee *_{\mathrm{v}}}^{m \vee I}=(-1)^{|I|(n-|I|)} \prod_{t \in \bullet_{\mathrm{v}}} A_{*_{\mathrm{v}} \vee t}^{m \vee I}=(-1)^{|I|(n-|I|)} \prod_{t \in \bullet_{\mathrm{v}}} A_{t \vee *_{\mathrm{v}}}^{I \vee m} .
\end{aligned}
$$

Proof. This is obvious by Lemma 4.1 along with

$$
\sigma(m \cup I, I \cup m)=1 \quad \text { and } \quad \sigma(m \vee I, I \vee m)=(-1)^{|I|} .
$$

Lastly, we write down another version of formula that uses the joining union. The effect of making another choice of order can be computed easily by Lemma 4.8. Here we use $m \vee I$ instead of $I \vee m$ in Theorem 4.6 and the effect is summarized via swapping $\frac{|I|(|I|+1)}{2}$ for $\frac{|I|(|I|-1)}{2}+n|I|$.

THEOREM 4.9. Under the same hypotheses as Theorem 4.6,

$$
\operatorname{vol}\left([0,1]^{n} \cap H^{+}\right)=\sum_{I \subset[m-1]} \sum_{\mathbf{v} \in F^{|I|} \cap H_{I}} \frac{(-1)^{\left|0_{\mathbf{v}}\right|+\frac{|I|(|I|-1)}{2}+n|I|}\left(g_{m}(\mathbf{v}) A_{*_{\mathrm{v}}}^{I}\right)^{n}}{n !\left|A_{*_{\mathrm{v}}}^{I}\right| \prod_{t \in I} A_{*_{\mathrm{v}}}^{I \vee \backslash t} \prod_{t \in \bullet_{\mathrm{v}}} A_{*_{\mathrm{v}} \vee t}^{m \vee I}} .
$$

4.4. Proof of the volume formula for clipped hypercubes. In this section, we prove Theorem 4.6. The other formulas of Theorem 4.5 and Theorem 4.9 can be derived from it as discussed in the previous section. For effective bookkeeping of complicated sign permutations, we introduce the separating parity $\Delta(I, J)$ of two indices $I \supset J$ as follows,

$$
\Delta(I, J):=\sigma(I,(I \backslash J) \vee J) .
$$

From the definition, we have the following lemmas about $\Delta$. 
Electronic Journal of Linear Algebra, ISSN 1081-3810

LEMMA 4.10. For any $I \subseteq[n]$,

$$
\Delta([n], I)=(-1)^{n|I|-\|I\|-\frac{|I|(|I|-1)}{2}} .
$$

In particular, for $t \in[n]$

$$
\Delta([n], t)=(-1)^{n-t}
$$

Proof. Let $I=\left\{i_{1}, \ldots, i_{|I|}\right\}$, then

$$
\begin{aligned}
{[n] \backslash I } & =\left\{1, \ldots, i_{1}-1, \widehat{i_{1}}, i_{1}+1, \ldots, i_{|I|}-1, \widehat{i_{|I|}}, i_{|I|}+1, \ldots, n\right\} \\
([n] \backslash I) \vee I & =\left\{1, \ldots, i_{1}-1, i_{1}+1 \ldots, i_{|I|}-1, i_{|I|}+1, \ldots, n\right\} \vee\left\{i_{1}, \ldots, i_{|I|}\right\} .
\end{aligned}
$$

We just count the number of transpositions. In order to shift each $i_{t}$ in $([n] \backslash I) \vee I$ into its original position in $[n]$, it requires $n-i_{t}-(|I|-t)$ transpositions.

$$
\sum_{t \in[|I|]} n-i_{t}-(|I|-t)=n|I|-\|I\|-\frac{|I|(|I|-1)}{2} .
$$

LEMMA 4.11. For any $I \subseteq[n]$,

$$
\prod_{i \in I} \Delta(I, i)=(-1)^{\frac{|I|(|I|-1)}{2}} .
$$

Proof. Let $I=\left\{i_{1}, i_{1}, \ldots, i_{|I|}\right\}$. Each $i_{t}=i$ requires $|I|-t$ transpositions. Hence,

$$
(-1)^{|I|-1}(-1)^{|I|-2} \cdots(-1)^{|I|-|I|}=(-1)^{\frac{|I|(|I|-1)}{2}} .
$$

At the first step of the proof, the summation over $\mathcal{I}$ and $\mathcal{H}_{\mathcal{I}}$ applied to Theorem 3.2 is converted into a summation over $I$ and $H_{I}$ as follows.

$$
\begin{aligned}
& \sum_{\substack{\mathcal{I} \subset[2 n+m-1] \\
|\mathcal{I}|=n}} \sum_{\substack{\mathbf{v} \in \mathcal{H}_{\mathcal{I}} \\
\mathcal{I}_{01} \vee \mathcal{I}_{*} \subset[2 n+m-1] \\
\left|\mathcal{I}_{01} \vee \mathcal{I}_{*}\right|=n}} \sum_{\substack{\mathbf{v} \in \mathcal{H}_{\mathcal{I}_{01}} \cap \mathcal{H}_{\mathcal{I}_{*}}\\
}} \\
& =\sum_{\mathcal{I}_{*} \subset[2 n+m-1] \backslash[2 n]} \sum_{\substack{\mathcal{I}_{01} \subset[2 n] \\
\left|\mathcal{I}_{01}\right|+\left|\mathcal{I}_{*}\right|=n}} \sum_{\mathbf{v} \in \mathcal{H}_{\mathcal{I}_{01}} \cap \mathcal{H}_{\mathcal{I}_{*}}} \\
& =\sum_{\mathcal{I}_{*} \subset[m-1]+2 n} \sum_{\mathbf{v} \in F^{\left|\mathcal{I}_{*}\right| \cap \mathcal{H}_{\mathcal{I}_{*}}}} \\
& =\sum_{I \subset[m-1]} \sum_{\mathbf{v} \in F^{|I|} \cap H_{I}} \text {. }
\end{aligned}
$$

For the sake of convenience, let $\Delta$ denote the separating parity of $[n]$ and $*_{\mathbf{v}}$, i.e.,

$$
\Delta:=\Delta\left([n], *_{\mathbf{v}}\right)=\sigma\left(\bullet_{\mathbf{v}} \cup *_{\mathbf{v}}, \bullet_{\mathbf{v}} \vee *_{\mathbf{v}}\right)
$$

Then we derive several relations between minors of $A$ and $\mathcal{A}$.

Proposition 4.12.

$$
\mathcal{A}_{[n]}^{\mathcal{I}}=(-1)^{\left|1_{\mathbf{v}}\right|} \Delta A_{*_{\mathbf{v}}}^{I}
$$


Proof. By Lemma 4.2 and (4.12),

$$
\begin{aligned}
& \mathcal{A}_{[n]}^{\mathcal{I}}=\mathcal{A}_{\bullet_{\mathrm{v}} \cup *_{\mathrm{v}}}^{\mathcal{I}_{01} \vee \mathcal{I}_{*}}=\Delta \mathcal{A}_{\boldsymbol{\bullet}_{\mathrm{v}} \vee *_{\mathrm{v}}}^{\mathcal{I}_{01} \vee \mathcal{I}_{*}} \\
& =\Delta \mathcal{A}_{\bullet_{\mathrm{v}}}^{\mathcal{I}_{01}} \mathcal{A}_{* \mathrm{v}}^{\mathcal{I}_{*}}=(-1)^{\left|1_{\mathrm{v}}\right|} \Delta A_{* \mathrm{v}}^{I} .
\end{aligned}
$$

Recall Lemma 4.2 and (4.12); the matrix $\mathcal{A}_{[n]}^{\mathcal{I}_{01}}$ is diagonal and $\mathcal{A}_{[n]}^{\mathcal{I}_{*}}=A_{[n]}^{I}$. Hence, we need to decompose $\mathcal{A}_{[n]}^{\mathcal{I} \backslash t \cup m}$ into $\mathcal{I}_{01}$ and $\mathcal{I}_{*}$ as follows.

$$
\prod_{t \in \mathcal{I}} \mathcal{A}_{[n]}^{\mathcal{I} \backslash t \cup m}=\prod_{t \in \mathcal{I}_{01}} \mathcal{A}_{[n]}^{\left(\mathcal{I}_{01} \backslash t\right) \cup \mathcal{I}_{*} \cup m} \prod_{t \in \mathcal{I}_{*}} \mathcal{A}_{[n]}^{\mathcal{I}_{01} \cup\left(\mathcal{I}_{*} \backslash t\right) \cup m} .
$$

The case of $\mathcal{I}_{*} \backslash t$ is proved in the same way.

Proposition 4.13.

$$
\prod_{t \in \mathcal{I}_{*}} \mathcal{A}_{[n]}^{\mathcal{I}_{01} \vee\left(\mathcal{I}_{*} \backslash t \vee m\right)}=(-1)^{|I|\left|1_{\mathbf{v}}\right|} \Delta^{|I|} \prod_{t \in I} A_{*_{\mathrm{v}}}^{I \backslash \vee \vee m}
$$

The case $\mathcal{I}_{01} \backslash t$ is much more complicated than the previous case.

Proposition 4.14.

$$
\prod_{t \in \mathcal{I}_{01}} \mathcal{A}_{[n]}^{\left(\mathcal{I}_{01} \backslash t\right) \vee \mathcal{I}_{*} \vee m}=(-1)^{\left|*_{\mathbf{v}} \|\right| 0_{\mathbf{v}}|+| 1_{\mathbf{v}}|| 0_{\mathbf{v}}|+| *_{\mathbf{v}}|| 1_{\mathbf{v}} \mid+\frac{\left|\bullet_{\mathbf{v}}\right|\left(\left|\bullet_{\mathbf{v}}\right|-1\right)}{2}} \Delta^{\left|\bullet_{\mathbf{v}}\right|} \prod_{t \in \bullet_{\mathbf{v}}} A_{*_{\mathrm{v}} \vee t}^{I_{*} \vee m} .
$$

Proof. First, divide $\mathcal{I}_{01}$ into $\mathcal{I}_{0}$ and $\mathcal{I}_{1}$, which correspond to $0_{\mathbf{v}}$ and $1_{\mathbf{v}}$ respectively.

$$
\begin{aligned}
& \prod_{t \in \mathcal{I}_{01}} \mathcal{A}_{[n]}^{\left(\mathcal{I}_{01} \backslash t\right) \vee \mathcal{I}_{*} \vee m}=\prod_{t \in \mathcal{I}_{01}} \mathcal{A}_{\bullet_{\mathrm{v}} \cup * \mathrm{v}}^{\left(\mathcal{I}_{01} \backslash t\right) \vee \mathcal{I}_{*} \vee m} \\
& =\prod_{t \in \mathcal{I}_{01}} \Delta \mathcal{A}_{\bullet_{\mathrm{v}} \vee *_{\mathrm{v}}}^{\left(\mathcal{I}_{0 \backslash \backslash t} \vee \mathcal{I}_{*} \vee m\right.} \\
& =\Delta^{\left|\mathcal{I}_{01}\right|} \prod_{t \in \mathcal{I}_{0}} \mathcal{A}_{\bullet_{\mathbf{v}} \vee *_{\mathrm{v}}}^{\left(\mathcal{I}_{01} \backslash t\right) \vee \mathcal{I}_{*} \vee m} \prod_{t \in \mathcal{I}_{1}} \mathcal{A}_{\boldsymbol{\bullet}_{\mathbf{v}} \vee * *_{\mathrm{v}}}^{\left(\mathcal{I}_{01} \backslash t\right) \vee \mathcal{I}_{*} \vee m} \\
& =\Delta^{\left|\mathcal{I}_{01}\right|} \prod_{t \in 0_{\mathrm{v}}} \mathcal{A}_{\bullet_{\mathrm{v}} \vee *_{\mathrm{v}}}^{\left(\mathcal{I}_{01} \backslash(2 t-1)\right) \vee \mathcal{I}_{*} \vee m} \prod_{t \in 1_{\mathrm{v}}} \mathcal{A}_{\bullet}^{\left(\mathcal{O}_{01} \backslash 2 t\right) \vee \mathcal{I}_{*} \vee \mathrm{v}}(.
\end{aligned}
$$

Each term is computed as follows.

$$
\begin{aligned}
& \prod_{t \in 0_{\mathbf{v}}} \mathcal{A}_{\bullet_{\mathfrak{v}} \vee *_{\mathbf{v}}}^{\left(\mathcal{I}_{01} \backslash(2 t-1)\right) \vee \mathcal{I}_{*} \vee m}=\prod_{t \in 0_{\mathbf{v}}} \Delta\left(\bullet_{\mathbf{v}}, t\right) \mathcal{A}_{\left(\bullet_{\mathbf{v}} \backslash t\right) \vee t \vee *_{\mathbf{v}}}^{\left(\mathcal{I}_{01} \backslash(2 t-1)\right) \vee \mathcal{I}_{*} \vee m} \\
& =(-1)^{\left|*_{\mathbf{v}}\right|\left|0_{\mathbf{v}}\right|} \prod_{t \in 0_{\mathbf{v}}} \Delta(\bullet \mathbf{v}, t) \mathcal{A}_{(\bullet \mathbf{v} \backslash t) \vee *_{\mathbf{v}} \vee t}^{\left(\mathcal{I}_{0} \backslash(2 t-1)\right) \vee \mathcal{I}_{*} \vee m} .
\end{aligned}
$$

By Lemma 4.2, we have $\mathcal{A}_{\bullet_{\mathbf{v}}}^{\mathcal{I}_{01}}=\left\{\begin{array}{cl}\mathcal{A}_{\bullet_{\mathbf{v}} \backslash t}^{\mathcal{I}_{01} \backslash(2 t-1)} & \text { if } t \in 0_{\mathbf{v}} \\ -\mathcal{A}_{\bullet_{\mathbf{v}} \backslash t}^{\mathcal{I}_{01} \backslash 2 t} & \text { if } t \in 1_{\mathbf{v}}\end{array}\right.$. Then,

$$
\begin{aligned}
& =(-1)^{\left|*_{\mathbf{v}} \| 0_{\mathbf{v}}\right|} \prod_{t \in 0_{\mathbf{v}}} \Delta\left(\bullet_{\mathbf{v}}, t\right) \mathcal{A}_{\bullet_{\mathbf{v}} \backslash t}^{\mathcal{I}_{01} \backslash(2 t-1)} A_{*_{\mathbf{v}} \vee t}^{\mathcal{I}_{*} \vee m} \\
& =(-1)^{\left|*_{\mathbf{v}} \| 0_{\mathbf{v}}\right|} \prod_{t \in 0_{\mathbf{v}}} \Delta\left(\bullet_{\mathbf{v}}, t\right)(-1)^{\left|1_{\mathbf{v}}\right|} \mathcal{A}_{*_{\mathbf{v}} \vee t}^{\mathcal{I}_{*} \vee m} \\
& =(-1)^{\left(\left|*_{\mathbf{v}}\right|+\left|1_{\mathbf{v}}\right|\right)\left|0_{\mathbf{v}}\right|} \prod_{t \in 0_{\mathbf{v}}} \Delta\left(\bullet_{\mathbf{v}}, t\right) \mathcal{A}_{*_{\mathbf{v}} \vee t}^{I_{*} \vee m}
\end{aligned}
$$


Similarly,

$$
\prod_{t \in 1_{\mathbf{v}}} \mathcal{A}_{\bullet_{\mathbf{v}} \vee *_{\mathbf{v}}}^{\left(\mathcal{I}_{01} \backslash 2 t\right) \vee \mathcal{I}_{*} \vee m}=(-1)^{\left(\left|*_{\mathbf{v}}\right|+\left|1_{\mathbf{v}}\right|\right)\left|1_{\mathbf{v}}\right|-\left|1_{\mathbf{v}}\right|} \prod_{t \in 1_{\mathbf{v}}} \Delta(\bullet \mathbf{v}, t) A_{*_{\mathbf{v}} \vee t}^{I_{*} \vee m}
$$

Take (4.17) and (4.18) together to complete the proof.

$$
\begin{aligned}
\prod_{t \in \mathcal{I}_{01}} \mathcal{A}_{[n]}^{\left(\mathcal{I}_{01} \backslash t\right) \vee \mathcal{I}_{*} \vee m} & =(-1)^{\left(\left|*_{\mathbf{v}}\right|+\left|1_{\mathbf{v}}\right|\right)\left(\left|0_{\mathbf{v}}\right|+\left|1_{\mathbf{v}}\right|\right)-\left|1_{\mathbf{v}}\right|} \Delta^{\left|\mathcal{I}_{01}\right|} \prod_{t \in \bullet_{\mathbf{v}}} \Delta\left(\bullet_{\mathbf{v}}, t\right) A_{*_{\mathbf{v}} \vee t}^{I_{*} \vee m} \\
& =(-1)^{\left|*_{\mathbf{v}}\right|\left|0_{\mathbf{v}}\right|+\left|1_{\mathbf{v}}\right|\left|0_{\mathbf{v}}\right|+\left|*_{\mathbf{v}}\right|\left|1_{\mathbf{v}}\right|+\frac{|\bullet \mathbf{v}|\left(\left|\bullet_{\mathbf{v}}\right|-1\right)}{2}} \Delta^{\left|\bullet_{\mathbf{v}}\right|} \prod_{t \in \bullet_{\mathbf{v}}} A_{*_{\mathbf{v}} \vee t}^{I_{*} \vee m}
\end{aligned}
$$

We put the three propositions $4.12,4.13$ and 4.14 together.

$$
\begin{aligned}
& \sum_{\substack{\mathcal{I} \subset[m-1] \\
|\mathcal{I}|=n}} \sum_{\mathbf{v} \in \mathcal{H}_{\mathcal{I}}} \frac{(-1)^{\frac{n(n+1)}{2}}\left(g_{m}(\mathbf{v}) \mathcal{A}_{[n]}^{\mathcal{I}}\right)^{n}}{n !\left|\mathcal{A}_{[n]}^{\mathcal{I}}\right| \prod_{t \in \mathcal{I}} \mathcal{A}_{[n]}^{\mathcal{I} \backslash t \cup m}}
\end{aligned}
$$

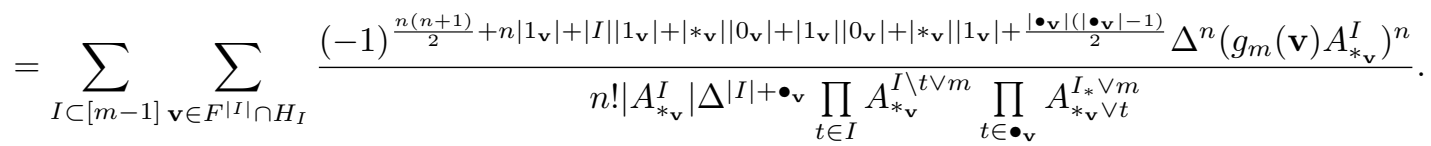

We calculate the parity expression,

$$
\begin{aligned}
& \frac{n(n+1)}{2}+n\left|1_{\mathbf{v}}\right|+|I|\left|1_{\mathbf{v}}\right|+\left|*_{\mathbf{v}}\right|\left|0_{\mathbf{v}}\right|+\left|1_{\mathbf{v}}\right|\left|0_{\mathbf{v}}\right|+\left|*_{\mathbf{v}}\right|\left|1_{\mathbf{v}}\right|+\frac{\left|\bullet_{\mathbf{v}}\right|\left(\left|\bullet_{\mathbf{v}}\right|-1\right)}{2} \\
& \underset{(\bmod 2)}{\equiv} \frac{n(n+1)}{2}+\frac{\left|\bullet_{\mathbf{v}}\right|\left(\left|\bullet_{\mathbf{v}}\right|+1\right)}{2}-\left|\bullet_{\mathbf{v}}\right|+n\left|1_{\mathbf{v}}\right|+\left|*_{\mathbf{v}}\right|\left|0_{\mathbf{v}}\right|+\left|1_{\mathbf{v}}\right|\left|0_{\mathbf{v}}\right| \\
& \underset{(\bmod 2)}{\equiv} \frac{\left|*_{\mathbf{v}}\right|\left(\left|*_{\mathbf{v}}\right|+1\right)}{2}+\left|*_{\mathbf{v}}\right|\left|\bullet_{\mathbf{v}}\right|+\left|\bullet_{\mathbf{v}}\right|+\left(n+\left|0_{\mathbf{v}}\right|\right)\left|1_{\mathbf{v}}\right|+\left|*_{\mathbf{v}}\right|\left|0_{\mathbf{v}}\right| \\
& \underset{(\bmod 2)}{\equiv} \frac{\left|*_{\mathbf{v}}\right|\left(\left|*_{\mathbf{v}}\right|+1\right)}{2}+\left|*_{\mathbf{v}}\right|\left|1_{\mathbf{v}}\right|+\left|\bullet_{\mathbf{v}}\right|+\left(\left|*_{\mathbf{v}}\right|+\left|1_{\mathbf{v}}\right|\right)\left|1_{\mathbf{v}}\right| \\
& \underset{(\bmod 2)}{\equiv} \frac{\left|*_{\mathbf{v}}\right|\left(\left|*_{\mathbf{v}}\right|+1\right)}{2}+\left|0_{\mathbf{v}}\right| .
\end{aligned}
$$

This completes the proof of Theorem 4.6.

5. More explicit formulas for $m \leq 3$. For the case of a small number of hyperplanes, we derive more concrete formulas in a fully explicit way. We expect for these formulas to be more accessible for a broader range of readers. Furthermore such an elementary and precise formulations play a crucial role in obtaining combinatorial identities in Section 6.

5.1. The case of at most two hyperplanes. First, let us consider only one halfspace, $m=1$. As we mention before, this case has been considered in the literature several times. The halfspace,

$$
H_{1}^{+}=\left\{\mathbf{x} \in \mathbb{R}^{n} \mid \mathbf{a} \cdot \mathbf{x}+r_{1}=a_{1} x_{1}+a_{2} x_{2}+\cdots+a_{n} x_{n}+r_{1} \geq 0\right\}
$$

is an auxiliary plane itself. We get $*_{\mathbf{v}}=\varnothing, I=\varnothing$ and $\left\|*_{\mathbf{v}}\right\|=|I|=0, A_{\varnothing}^{\varnothing}=1$ and $A_{i}^{1}=a_{i}$. The good clipping condition $(\mathrm{A})$ automatically holds and $(\mathrm{B})$ is equivalent to $\prod_{t=1}^{n} a_{t} \neq 0$. Applying these terms to Theorem 4.5 we get a proof of Theorem 1.1. 
Second, let us prove Corollary 1.2. Consider the following two hyperplanes,

$$
\begin{aligned}
& H_{1}^{+}=\left\{\mathbf{x} \in \mathbb{R}^{n} \mid \mathbf{a} \cdot \mathbf{x}+r_{1}=a_{1} x_{1}+a_{2} x_{2}+\cdots+a_{n} x_{n}+r_{1} \geq 0\right\}, \\
& H_{2}^{+}=\left\{\mathbf{x} \in \mathbb{R}^{n} \mid \mathbf{b} \cdot \mathbf{x}+r_{2}=b_{1} x_{1}+b_{2} x_{2}+\cdots+b_{n} x_{n}+r_{2} \geq 0\right\}
\end{aligned}
$$

We see that $*_{\mathbf{v}}$ and $I$ become the empty set or a set of only one element. The former case of the empty set is the same as the "one hyperplane" case. For the case of $I=[1]=\{1\}$, we put $*_{\mathbf{v}}=\{*(\mathbf{v})\}$ then $\bullet_{\mathbf{v}}=[n] \backslash * \mathbf{v}$ and get

$$
\prod_{t \in \bullet \mathbf{v}} A_{* \mathbf{v} \vee t}^{I \vee m}=\prod_{t \in \bullet \mathbf{v}}\left|\begin{array}{cc}
a_{*(\mathbf{v})} & b_{*(\mathbf{v})} \\
a_{t} & b_{t}
\end{array}\right| .
$$

Applying Theorem 4.6 to these, we obtain Corollary 1.2. Here, the good clipping conditions are

(A) $F^{0} \cap H_{1} \cap H_{2}^{+}=\varnothing$,

(B) $\prod_{t=1}^{n} b_{t} \prod_{t \in \bullet \mathbf{v}}\left|\begin{array}{cc}a_{*(\mathbf{v})} & b_{*(\mathbf{v})} \\ a_{t} & b_{t}\end{array}\right| \neq 0 \quad$ for $\mathbf{v} \in F^{1} \cap H_{1} \cap H_{2}^{+}$.

5.2. The case of three hyperplanes. Let us consider three halfspaces, $m=3$. We formulate this case in a similar fashion to the one or two hyperplane cases, in particular, which is important to derive several identities in Appendix C.

COROLlary 5.1. The volume of the standard unit hypercube $[0,1]^{n}$ intersecting the three halfspaces

$$
\begin{aligned}
& H_{1}^{+}=\left\{\mathbf{x} \in \mathbb{R}^{n} \mid \mathbf{a} \cdot \mathbf{x}+r_{1}=a_{1} x_{1}+a_{2} x_{2}+\cdots+a_{n} x_{n}+r_{1} \geq 0\right\}, \\
& H_{2}^{+}=\left\{\mathbf{x} \in \mathbb{R}^{n} \mid \mathbf{b} \cdot \mathbf{x}+r_{2}=b_{1} x_{1}+b_{2} x_{2}+\cdots+b_{n} x_{n}+r_{2} \geq 0\right\} \\
& H_{3}^{+}=\left\{\mathbf{x} \in \mathbb{R}^{n} \mid \mathbf{c} \cdot \mathbf{x}+r_{3}=c_{1} x_{1}+c_{2} x_{2}+\cdots+c_{n} x_{n}+r_{3} \geq 0\right\}
\end{aligned}
$$

satisfying good clipping conditions, is

$$
\begin{aligned}
& \operatorname{vol}\left([0,1]^{n} \cap H_{1}^{+} \cap H_{2}^{+} \cap H_{3}^{+}\right)=\sum_{\mathbf{v} \in F^{0} \cap H_{1}^{+} \cap H_{2}^{+} \cap H_{3}^{+}} \frac{(-1)^{\left|0_{\mathbf{v}}\right|} g_{3}(\mathbf{v})^{n}}{n ! \prod_{t \in[n]} c_{t}} \\
& -\sum_{\mathbf{v} \in F^{1} \cap H_{1} \cap H_{2}^{+} \cap H_{3}^{+}} \frac{(-1)^{\left|0_{\mathbf{v}}\right|} \operatorname{sgn}\left(a_{*(\mathbf{v})}\right) a_{*(\mathbf{v})}^{n-1} g_{3}(\mathbf{v})^{n}}{n ! c_{*(\mathbf{v})} \prod_{t \in \bullet \mathbf{v}}\left|\begin{array}{cc}
a_{*(\mathbf{v})} & c_{*(\mathbf{v})} \\
a_{t} & c_{t}
\end{array}\right|} \\
& -\sum_{\mathbf{v} \in F^{1} \cap H_{1}^{+} \cap H_{2} \cap H_{3}^{+}} \frac{(-1)^{\left|0_{\mathbf{v}}\right|} \operatorname{sgn}\left(b_{*(\mathbf{v})}\right) b_{*(\mathbf{v})}^{n-1} g_{3}(\mathbf{v})^{n}}{n ! c_{*(\mathbf{v})} \prod_{t \in \bullet \mathbf{v}}\left|\begin{array}{ccc}
b_{*(\mathbf{v})} & c_{*(\mathbf{v})} \\
b_{t} & c_{t}
\end{array}\right|} \\
& -\sum_{\substack{\mathbf{v} \in F^{2} \cap \\
H_{1} \cap H_{2} \cap H_{3}^{+}}} \frac{(-1)^{\left|0_{\mathbf{v}}\right|} \operatorname{sgn}\left(\left|\begin{array}{ll}
a_{*_{1}(\mathbf{v})} & b_{*_{1}(\mathbf{v})} \\
a_{*_{2}(\mathbf{v})} & b_{*_{2}(\mathbf{v})}
\end{array}\right|\right)\left|\begin{array}{cc}
a_{*_{1}(\mathbf{v})} & b_{*_{1}(\mathbf{v})} \\
a_{*_{2}(\mathbf{v})} & b_{*_{2}(\mathbf{v})}
\end{array}\right|^{n-1} g_{3}(\mathbf{v})^{n}}{\left|\begin{array}{cc|c|c|c|cc}
a_{*_{1}(\mathbf{v})} & c_{*_{1}(\mathbf{v})} \\
a_{*_{2}(\mathbf{v})} & c_{*_{2}(\mathbf{v})}
\end{array}\right|\left|\begin{array}{ll}
b_{*_{1}(\mathbf{v})} & c_{*_{1}(\mathbf{v})} \\
b_{*_{2}(\mathbf{v})} & c_{*_{2}(\mathbf{v})}
\end{array}\right| \prod_{t \in \bullet_{\mathbf{v}}}\left|\begin{array}{ccc}
a_{*_{1}(\mathbf{v})} & b_{*_{1}(\mathbf{v})} & c_{*_{1}(\mathbf{v})} \\
a_{*_{2}(\mathbf{v})} & b_{*_{2}(\mathbf{v})} & c_{*_{2}(\mathbf{v})} \\
a_{t} & b_{t} & c_{t}
\end{array}\right|}
\end{aligned}
$$


Proof. For each vertex, $\left|*_{\mathbf{v}}\right|=|I|=0,1$, or 2 . The former two cases are the same as the case of fewer than two hyperplanes. Let us consider the $I=[2]$ case. Recall $\bullet_{\mathbf{v}}=[n] \backslash *_{\mathbf{v}}$ and $*_{\mathbf{v}}=\left\{*_{1}(\mathbf{v}), *_{2}(\mathbf{v})\right\}$ then

$$
\prod_{t \in I} A_{*_{\mathbf{v}}}^{I \vee m \backslash t}=\left|\begin{array}{ll}
a_{*_{1}(\mathbf{v})} & c_{*_{1}(\mathbf{v})} \\
a_{*_{2}(\mathbf{v})} & c_{*_{2}(\mathbf{v})}
\end{array}\right|\left|\begin{array}{cc}
b_{*_{1}(\mathbf{v})} & c_{*_{1}(\mathbf{v})} \\
b_{*_{2}(\mathbf{v})} & c_{*_{2}(\mathbf{v})}
\end{array}\right|
$$

and

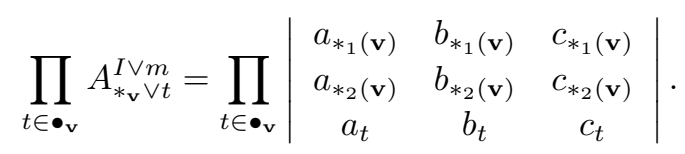

5.3. Examples of calculations. We show two examples of calculations using Corollary 1.2 and Corollary 5.1. In particular the following examples have several non-simple vertices. But we can apply our formulas to them because all non-simple vertices lie in the auxiliary hyperplane.

EXAmple 5.2. Let us calculate the volume of the clipped hypercube $[0,1]^{3}$ which intersects the following two halfspaces,

$$
\begin{aligned}
& H_{1}^{+}=\left\{\mathbf{x} \in \mathbb{R}^{n} \mid \mathbf{a} \cdot \mathbf{x}+\frac{1}{2}=-x_{1}+x_{2}+\frac{1}{2} \geq 0\right\}, \\
& H_{2}^{+}=\left\{\mathbf{x} \in \mathbb{R}^{n} \mid \mathbf{b} \cdot \mathbf{x}+3=-x_{1}-2 x_{2}-x_{3}+3 \geq 0\right\},
\end{aligned}
$$

Let us find the vertices of the clipped hypercube. There are five vertices in $F^{0} \cap H_{1}^{+} \cap H_{2}^{+}$:

$$
\mathbf{v}_{1}=(0,0,0), \quad \mathbf{v}_{2}=(0,0,1), \quad \mathbf{v}_{3}=(0,1,0), \quad \mathbf{v}_{4}=(0,1,1), \quad \mathbf{v}_{5}=(1,1,0)
$$

and four vertices in $F^{1} \cap H_{1} \cap H_{2}^{+}$:

$$
\mathbf{v}_{6}=\left(\frac{1}{2}, 0,0\right), \quad \mathbf{v}_{7}=\left(\frac{1}{2}, 0,1\right), \quad \mathbf{v}_{8}=\left(1, \frac{1}{2}, 0\right), \quad \mathbf{v}_{9}=\left(1, \frac{1}{2}, 1\right) .
$$

Among those vertices, $\mathbf{v}_{4}, \mathbf{v}_{5}$ and $\mathbf{v}_{9}$ lie on $H_{2}$ and we don't need to worry about these vertices. We can check that the good clipping conditions hold. We calculate the values $N_{\mathbf{v}_{i}}$ for $i=1,2,3,6,7,8$ by Corollary 1.2. For example, we have

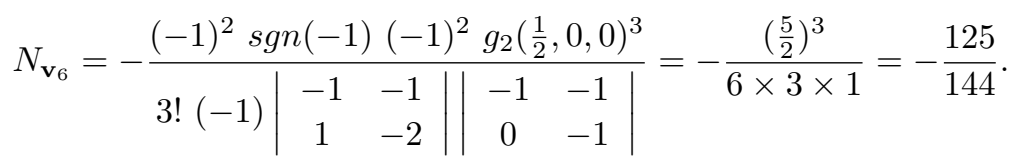

Therefore, we get

$$
\begin{aligned}
\operatorname{vol}\left([0,1]^{3} \cap H_{1}^{+} \cap H_{2}^{+}\right)= & \sum_{\mathbf{v} \in\left\{\mathbf{v}_{1}, \mathbf{v}_{2}, \mathbf{v}_{3}\right\}} \frac{(-1)^{\left|0_{\mathbf{v}}\right|} g_{2}(\mathbf{v})^{3}}{3 ! \prod_{t=1}^{3} b_{t}} \\
& -\sum_{\mathbf{v} \in\left\{\mathbf{v}_{6}, \mathbf{v}_{7}, \mathbf{v}_{8}\right\}} \frac{(-1)^{\left|0_{\mathbf{v}}\right|} a_{*(\mathbf{v})}^{3} g_{2}(\mathbf{v})^{3}}{3 !\left|a_{*(\mathbf{v})}\right|} b_{*(\mathbf{v})} \prod_{t \in[3] \backslash *(\mathbf{v})}\left|\begin{array}{cc}
a_{*(\mathbf{v})} & b_{*(\mathbf{v})} \\
a_{t} & b_{t}
\end{array}\right| \\
= & \frac{9}{4}-\frac{2}{3}-\frac{1}{12}-\frac{125}{144}+\frac{27}{144}-\frac{1}{36} \\
= & \frac{19}{24} .
\end{aligned}
$$


REMARK 5.3. Note that the polyhedron $[0,1]^{3} \cap H_{1}^{+} \cap H_{2}^{+}$has three non-simple vertices $\mathbf{v}_{4}, \mathbf{v}_{5}, \mathbf{v}_{9}$ but all these are degenerate into $\mathrm{H}_{2}$ so we can apply the formula of Corollary 1.2. Notice that if one changes the roles of the two halfspaces then one cannot apply the formula, because there are non-degenerate non-simple vertices and it violates the good clipping conditions.

EXAMPLE 5.4. Let us calculate the volume of the region of $[0,1]^{3}$ that intersects the three halfspaces,

$$
\begin{aligned}
& H_{1}^{+}=\left\{x \mid-x_{1}+x_{2}+\frac{1}{2} \geq 0\right\}, \\
& H_{2}^{+}=\left\{x \mid x_{3}-\frac{1}{2} \geq 0\right\}, \\
& H_{3}^{+}=\left\{x \mid-x_{1}-2 x_{2}-x_{3}+3 \geq 0\right\} .
\end{aligned}
$$

The three halfspaces satisfy the good clipping conditions and we can apply Corollary 5.1. Let us find vertices for each $I \subset[3-1]$, i.e., for $I=\varnothing,\{1\},\{2\}$ and $\{1,2\}$ :

$$
\begin{array}{ll}
F^{0} \cap H_{1}^{+} \cap H_{2}^{+} \cap H_{3}^{+}: & \mathbf{v}_{1}=(0,0,1), \mathbf{v}_{2}=(0,1,1), \\
F^{1} \cap H_{1} \cap H_{2}^{+} \cap H_{3}^{+}: & \mathbf{v}_{3}=\left(\frac{1}{2}, 0,1\right), \mathbf{v}_{4}=\left(1, \frac{1}{2}, 1\right), \\
F^{1} \cap H_{1}^{+} \cap H_{2} \cap H_{3}^{+}: & \mathbf{v}_{5}=\left(0,0, \frac{1}{2}\right), \mathbf{v}_{6}=\left(0,1, \frac{1}{2}\right), \\
F^{2} \cap H_{1} \cap H_{2} \cap H_{3}^{+}: & \mathbf{v}_{7}=\left(\frac{1}{2}, 0, \frac{1}{2}\right), \mathbf{v}_{8}=\left(1, \frac{1}{2}, \frac{1}{2}\right) .
\end{array}
$$

Let us check that $\mathbf{v}_{2}$ and $\mathbf{v}_{4}$ lie on $H_{3}$ and these vertices are degenerate vertices. Note that there are two more degenerate simple vertices $\mathbf{v}_{9}=\left(1, \frac{3}{4}, \frac{1}{2}\right)$ and $\mathbf{v}_{10}=\left(\frac{1}{2}, 1, \frac{1}{2}\right)$ which are excluded from the summation. In summary, the polyhedron $[0,1]^{3} \cap H_{1}^{+} \cap H_{2}^{+} \cap H_{3}^{+}$has ten vertices with two non-simple vertices $\mathbf{v}_{2}$ and $\mathbf{v}_{4}$ among them. After applying Corollary 5.1 to these, we obtain

$$
N_{\mathbf{v}_{1}}=-\frac{2}{3}, \quad N_{\mathbf{v}_{3}}=\frac{3}{16}, \quad N_{\mathbf{v}_{5}}=\frac{125}{96}, \quad N_{\mathbf{v}_{6}}=-\frac{1}{96}, \quad N_{\mathbf{v}_{7}}=-\frac{4}{9}, \quad \text { and } \quad N_{\mathbf{v}_{8}}=-\frac{1}{288} .
$$

Therefore, we obtain the volume as follows,

$$
\begin{aligned}
\operatorname{vol}\left([0,1]^{3} \cap H_{1}^{+} \cap H_{2}^{+} \cap H_{3}^{+}\right) & =\sum_{\mathbf{v}=\mathbf{v}_{1}} N_{\mathbf{v}}+\sum_{\mathbf{v}=\mathbf{v}_{3}} N_{\mathbf{v}}+\sum_{\mathbf{v} \in\left\{\mathbf{v}_{5}, \mathbf{v}_{6}\right\}} N_{\mathbf{v}}+\sum_{\mathbf{v} \in\left\{\mathbf{v}_{7}, \mathbf{v}_{8}\right\}} N_{\mathbf{v}} \\
& =-\frac{2}{3}+\frac{3}{16}+\frac{125}{96}-\frac{1}{96}-\frac{4}{9}-\frac{1}{288} \\
& =\frac{35}{96} .
\end{aligned}
$$

\section{Combinatorial identities from clipping hypercubes.}

6.1. From polytopes to identities. Let us describe a general method to produce a combinatorial identity from a polytope volume. This is a simple observation that the resulting volume is independent of the choice of an auxiliary plane. Recall the volume expression of Theorem 3.2 and theorems in Section 4.3 and let us assume that we already know the volume of a clipped hypercube $P=[0,1]^{n} \cap H_{1}^{+} \cap \cdots \cap H_{m-1}^{+}$. Let us cut $P$ into two pieces one more time as

$$
P_{+}=P \cap H_{m}^{+} \quad \text { and } \quad P_{-}=P \cap H_{m}^{-}
$$

by the auxiliary hyperplane $H_{m}=\left\{a_{1} x_{1}+\cdots+a_{n} x_{n}+y=0\right\}$. No matter how we take $H_{m}$, the union of two pieces should be $P$ and

$$
\operatorname{vol}\left(P_{+}\right)+\operatorname{vol}\left(P_{-}\right)=\operatorname{vol}(P)
$$


The known volume is constant and is expressed in terms of the free variables $a_{1}, a_{2}, \ldots, a_{n}$ and $y$ of the coefficients of $H_{m}$. They will produce an algebraic identity.

REMARK 6.1. Only hyperplanes satisfying good clipping conditions actually produce an algebraic identity since the volume formula makes sense only for this case. However, as we discussed in Section 3.4, we can use a limiting argument since the volume function is continuous function and the good clipping conditions are open conditions. Therefore, whether a good clipping condition is satisfied or not, the resulting algebraic identity holds as long as the expression is valid.

REMARK 6.2. The volume formulas are homogeneous for $a_{1}, a_{2}, \ldots a_{n}$ because they are composed of homogeneous polynomials which are determinants of matrices with one column vector of indeterminate $\mathbf{a}=\left(a_{1}, a_{2}, \ldots, a_{n}\right)$.

Let us see the most simple case which is a direct consequence of Theorem 1.1.

Corollary 6.3. Let $\mathbf{a}=\left(a_{1}, \ldots, a_{n}\right) \in \mathbb{R}^{n}$ and $y \in \mathbb{R}$. Then

$$
\sum_{\mathbf{v} \in F^{0}}(-1)^{\left|0_{\mathbf{v}}\right|}(\mathbf{a} \cdot \mathbf{v}+y)^{n}=n ! a_{1} a_{2} \cdots a_{n} .
$$

Proof. For a hyperplane

$$
H_{1}=\left\{\mathbf{x} \mid g(\mathbf{x}):=\mathbf{a} \cdot \mathbf{x}+y=a_{1} x_{1}+\cdots+a_{n} x_{n}+y=0\right\},
$$

we get

$$
\operatorname{vol}\left([0,1]^{n} \cap H_{1}^{-}\right)=\sum_{\mathbf{v} \in F^{0} \cap H_{1}^{-}} \frac{(-1)^{\left|0_{\mathbf{v}}\right|} g(\mathbf{v})^{n}}{n ! \prod_{t=1}^{n} a_{t}}
$$

and

$$
\operatorname{vol}\left([0,1]^{n}\right)=\operatorname{vol}\left([0,1]^{n} \cap H_{1}^{+}\right)+\operatorname{vol}\left([0,1]^{n} \cap H_{1}^{-}\right)
$$

so we have

$$
1=\sum_{\mathbf{v} \in F^{0}} \frac{(-1)^{\left|0_{\mathbf{v}}\right|} g(\mathbf{v})^{n}}{n ! \prod_{t=1}^{n} a_{t}}
$$

Finally, we get

$$
\sum_{\mathbf{v} \in F^{0}}(-1)^{\left|0_{\mathbf{v}}\right|}(\mathbf{a} \cdot \mathbf{v}+y)^{n}=n ! \prod_{t=1}^{n} a_{t} .
$$

Let us consider the summation over $\mathbf{v} \in F^{0}=\{0,1\}^{n}$. We can replace this summation by $1 \leq$ $t_{1}, \ldots t_{i} \leq n$ as regarding $1_{\mathbf{v}}=\left\{t_{1}, \ldots, t_{i}\right\}$ and hence prove Theorem 1.3. Note that $a_{i}$ should be non-zero when applying the volume formula but the resulting identity has no such constraint by continuity, as we remarked above.

Essentially, whenever we take a polytope, we can find a corresponding combinatorial identity if we have a concrete volume formula. Hence, we can expect this kind of

$$
\{\text { polytopes }\} \longrightarrow\{\text { combinatorial identities }\}
$$

correspondence has a structural property. At this stage, it seems to be somewhat vague to investigate the resulting identities from general convex polytopes. Here, we present a few cases. We give the case of a clipped hypercube by a symmetric hyperplane with full generality in the next section, which produces the interesting identity in Theorem 1.6. We treat several more examples of resulting identities in the Appendix. 
6.2. Symmetric arrangements of hyperplanes. When we see the identity of Theorem 1.3 , we can observe that this is a symmetric function of the $n$-variables $a_{1}, \ldots a_{n}$. This property comes from the fact that the polytope itself under consideration is symmetric, i.e., we took a symmetric arrangement of hyperplanes, where the term symmetric means that hyperplanes except the auxiliary hyperplane are invariant under exchange of coordinate axes of $\mathbb{R}^{n}$.

Probably the second easiest example of a symmetric arrangement is

$$
H_{1}^{+}=\left\{\mathbf{x} \in \mathbb{R}^{n} \mid-x_{1}-x_{2}-\cdots-x_{n}+1 \geq 0\right\} .
$$

We use the auxiliary hyperplane

$$
H_{2}=\left\{\mathbf{x} \in \mathbb{R}^{n} \mid a_{1} x_{1}+\cdots a_{n} x_{n}+y=0\right\}
$$

in the formula of the $m=2$ case and obtain the identity of Theorem 1.4. We remark that the identity of Theorem 1.4 is a direct consequence of Proposition 1 in [2].

Note that $H_{1}$ and $H_{2}$ violate the good clipping conditions. So we use the $\epsilon$-perturbation proposed in Section 4.2 when applying the volume formula.

Proof of Theorem 1.4. For sufficiently small $\epsilon>0$, the polytope $[0,1]^{n} \cap H_{1}^{+}$with

$$
H_{1}^{+}=\left\{\mathbf{x} \mid-x_{1}-x_{2}-\cdots-x_{n}+1-\epsilon \geq 0\right\}
$$

satisfies the good clipping conditions. We have

$$
\begin{aligned}
& \operatorname{vol}\left([0,1]^{n} \cap H_{1}^{+}\right)= \operatorname{vol}\left([0,1]^{n} \cap H_{1}^{+} \cap H_{2}^{+}\right)+\operatorname{vol}\left([0,1]^{n} \cap H_{1}^{+} \cap H_{2}^{-}\right) \\
&= \sum_{\mathbf{v} \in F^{0} \cap H_{1}^{+} \cap H_{2}^{+}}-\sum_{\mathbf{v} \in F^{1} \cap H_{1} \cap H_{2}^{+}}-\sum_{\mathbf{v} \in F^{0} \cap H_{1}^{+} \cap H_{2}^{-}} \\
&= \sum_{\mathbf{v} \in F^{0} \cap H_{1}^{+}} \frac{(-1)^{\left|0_{\mathbf{v}}\right|} g_{2}(\mathbf{v})^{n}}{n ! \prod_{t=1}^{n} a_{t}} \\
&-\sum_{\mathbf{v} \in F^{1} \cap H_{1}} \frac{(-1)^{-}}{n ! a_{*(\mathbf{v}} \mid}(-1)^{n} g_{2}(\mathbf{v})^{n} \\
& \prod_{t=1, t \neq *(\mathbf{v})}^{n}\left|\begin{array}{cc}
-1 & a_{*(\mathbf{v})} \\
-1 & a_{t}
\end{array}\right|
\end{aligned}
$$

Then there are $n+1$ vertices of

$$
\begin{gathered}
\mathbf{v}=(0,0, \ldots, 0) \in F^{0} \cap H_{1}^{+} \\
\mathbf{v}_{i}=(1-\epsilon) \mathbf{e}_{i} \in F^{1} \cap H_{1} \quad \text { for } i \in[n] .
\end{gathered}
$$

Hence, we obtain

$$
\frac{(1-\epsilon)^{n}}{n !}=\frac{(-1)^{n} y^{n}}{n ! a_{1} a_{2} \cdots a_{n}}-\sum_{i=1}^{n} \frac{(-1)^{n-1}(-1)^{n}\left(a_{i}(1-\epsilon)+y\right)^{n}}{n ! a_{i} \prod_{t=1, t \neq i}^{n}\left(a_{i}-a_{t}\right)}
$$

The result now follows by taking $\epsilon \rightarrow 0$ and simplifying.

We next consider the following half space,

$$
H_{1}^{+}=\left\{\mathbf{x} \in \mathbb{R}^{n} \mid-x_{1}-x_{2}-\cdots-x_{n}+2 \geq 0\right\} .
$$


Electronic Journal of Linear Algebra, ISSN 1081-3810

A publication of the International Linear Algebra Society

Volume 36, pp. 228-255, April 2020

By the same $\epsilon$-perturbation taking $2-\epsilon$ instead of 2 , we get the following identity,

$$
\frac{y^{n}}{a_{1} a_{2} \cdots a_{n}}-\sum_{i=1}^{n} \frac{\left(y+a_{i}\right)^{n}}{a_{1} a_{2} \cdots a_{n}}+\sum_{1 \leq t_{1}<t_{2} \leq n} \sum_{i=1}^{2} \frac{\left(y+a_{t_{1}}+a_{t_{2}}\right)^{n}}{a_{t_{i}}\left(a_{1}-a_{t_{i}}\right)\left(a_{2}-a_{t_{i}}\right) \cdots\left(a_{n}-a_{t_{i}}\right)}=(-1)^{n}\left(2^{n}-n\right)
$$

We consider all possible symmetric arrangements of only one hyperplane. Then all of the linear coefficients of $H_{1}$ should be the same. So it is reasonable to think about

$$
H_{1}^{+}=\left\{\mathbf{x} \in \mathbb{R}^{n} \mid-x_{1}-x_{2}-\cdots-x_{n}+l \geq 0\right\} \quad \text { for } l \in[n] .
$$

This gives the following theorem which is nothing but a different form of Theorem 1.6.

TheOREM 6.4. For an integer $l \in[n]$ and non-zero distinct real numbers $a_{1}, a_{2}, \ldots, a_{n}$ and $y \in \mathbb{R}$,

$$
\begin{aligned}
& \frac{y^{n}}{a_{1} a_{2} \cdots a_{n}}+\sum_{i=1}^{l-1} \sum_{1 \leq t_{1}<t_{2}<\cdots<t_{i} \leq n} \frac{(-1)^{i}\left(y+a_{t_{1}}+a_{t_{2}}+\cdots+a_{t_{i}}\right)^{n}}{a_{1} a_{2} \cdots a_{n}} \\
& +(-1)^{l} \sum_{1 \leq t_{1}<\cdots<t_{l} \leq n} \sum_{i=1}^{l} \frac{\left(y+a_{t_{1}}+\cdots+a_{t_{l}}\right)^{n}}{a_{t_{i}}\left(a_{1}-a_{t_{i}}\right)\left(a_{2}-a_{t_{i}}\right) \cdots\left(a_{n}-a_{t_{i}}\right)} \\
& =\sum_{i=0}^{l-1}(-1)^{n-i}\left(\begin{array}{c}
n \\
i
\end{array}\right)(l-i)^{n},
\end{aligned}
$$

or equivalently,

$$
\begin{aligned}
& \sum_{i=1}^{l-1} \sum_{1 \leq t_{1}<t_{2}<\cdots<t_{i} \leq n}(-1)^{i}\left(a_{t_{1}}+a_{t_{2}}+\cdots+a_{t_{i}}\right)^{k} \\
& +(-1)^{l} \sum_{1 \leq t_{1}<\cdots<t_{l} \leq n} \sum_{i=1}^{l}\left(\prod_{j=1, j \neq t_{i}}^{n} \frac{a_{j}}{a_{j}-a_{t_{i}}}\right)\left(a_{t_{1}}+\cdots+a_{t_{l}}\right)^{k} \\
& = \begin{cases}-1, & \text { if } k=0, \\
0, & \text { if } k=1,2, \ldots, n-1, \\
\prod_{i=1}^{n} a_{i} \sum_{i=0}^{l-1}(-1)^{n-i}\left(\begin{array}{c}
n \\
i
\end{array}\right)(l-i)^{n}, & \text { if } k=n,\end{cases}
\end{aligned}
$$

or, using set-notation with $A=\left\{a_{1}, a_{2}, \ldots, a_{n}\right\}$,

$$
\sum_{\substack{I \subset A \\
|I|<l}}(-1)^{|I|}(y+\|I\|)^{n}+\sum_{\substack{I \subset A \\
|I|=l}}(-1)^{l}(y+\|I\|)^{n}\left(\sum_{a \in I} \prod_{b \in A \backslash a} \frac{b}{b-a}\right)=A ! \sum_{i=0}^{l-1}(-1)^{n-i}\left(\begin{array}{c}
n \\
i
\end{array}\right)(l-i)^{n} .
$$

One needs to be careful here; there is a difference between Theorem 1.6 and the second form above in the case $k=0$ because $\|\varnothing\|^{0}=0^{0}=1$.

Proof. We use $\epsilon$-perturbation replacing $l$ by $l-\epsilon$ for $H_{1}$, then

$$
\operatorname{vol}\left([0,1]^{n} \cap H_{1}^{+}\right)=\sum_{\mathbf{v} \in F^{0} \cap H_{1}^{+}} \frac{(-1)^{\left|0_{\mathbf{v}}\right|}(\mathbf{a} \cdot \mathbf{v}+y)^{n}}{n ! a_{1} a_{2} \cdots a_{n}}-\sum_{\mathbf{v} \in F^{1} \cap H_{1}} \frac{(-1)^{n-l}(-1)^{n}(\mathbf{a} \cdot \mathbf{v}+y)^{n}}{n ! a_{*(\mathbf{v})} \prod_{t=1, t \neq *(\mathbf{v})}\left(a_{*(\mathbf{v})}-a_{t}\right)} .
$$


For $F^{0} \cap H_{1}^{+}$, there are a total of $\left(\begin{array}{c}n \\ 0\end{array}\right)+\left(\begin{array}{c}n \\ 1\end{array}\right)+\cdots+\left(\begin{array}{c}n \\ l-1\end{array}\right)$ vertices, i.e., there are $l$ families of vertices with respect to the sum of the coordinate values of $\mathbf{v}$. Also, for $F^{1} \cap H_{1}$, there are $l\left(\begin{array}{c}n \\ l\end{array}\right)$ vertices whose coordinate values are $l-1$ ones, a unique $1-\epsilon$, and $n-l$ zeros. Hence, we obtain

$$
\sum_{\mathbf{v} \in F^{0} \cap H_{1}^{+}} \frac{(-1)^{\left|0_{\mathbf{v}}\right|}(\mathbf{a} \cdot \mathbf{v}+y)^{n}}{n ! a_{1} a_{2} \cdots a_{n}}=\frac{(-1)^{n} y^{n}}{n ! a_{1} a_{2} \cdots a_{n}}+\sum_{i=1}^{l-1} \sum_{1 \leq t_{1}<\cdots<t_{i} \leq n} \frac{(-1)^{n-i}\left(y+a_{t_{1}}+\cdots+a_{t_{i}}\right)^{n}}{n ! a_{1} a_{2} \cdots a_{n}}
$$

and

$$
\sum_{\mathbf{v} \in F^{1} \cap H_{1}} \frac{(-1)^{l+1}(\mathbf{a} \cdot \mathbf{v}+y)^{n}}{n ! a_{*(\mathbf{v})} \prod_{t=1, t \neq *(\mathbf{v})}\left(a_{*(\mathbf{v})}-a_{t}\right)}=(-1)^{l+1} \sum_{1 \leq t_{1}<\cdots<t_{l} \leq n} \sum_{i=1}^{l} \frac{\left(y+a_{t_{1}}+\cdots+a_{t_{i}}(1-\epsilon)+\cdots+a_{t_{l}}\right)^{n}}{n ! a_{t_{i}}\left(a_{t_{i}}-a_{1}\right)\left(a_{t_{i}}-a_{2}\right) \cdots\left(a_{t_{i}}-a_{n}\right)}
$$

We compute the volume of the clipped hypercube using Theorem 1.1,

$$
\begin{aligned}
\operatorname{vol}\left([0,1]^{n} \cap H_{1}^{+}\right)= & \frac{(-1)^{n}(l-\epsilon)^{n}}{n !(-1)^{n}}+\frac{(-1)^{n-1}\left(\begin{array}{c}
n \\
1
\end{array}\right)(l-1-\epsilon)^{n}}{n !(-1)^{n}}+\cdots \\
& +\frac{(-1)^{n-(l-1)}\left(\begin{array}{c}
n \\
l-1
\end{array}\right)(l-(l-1)-\epsilon)^{n}}{n !(-1)^{n}} \\
= & \sum_{i=0}^{l-1}(-1)^{i}\left(\begin{array}{c}
n \\
i
\end{array}\right) \frac{(l-i-\epsilon)^{n}}{n !} .
\end{aligned}
$$

By taking $\epsilon \rightarrow 0$, we obtain the result.

REMARK 6.5. If we take $l$ to be a non-integer real number, we get a slightly different identity, obtained by rescaling variables from the result of Theorem 6.4. If the vertex configuration is preserved under changing hyperplanes, the resulting identity is essentially the same as the previous one.

\section{Appendix. Several clipped hypercube identities.}

For simplicity, we do not use $m$ for the number of hyperplanes in the appendix section and use $\mathbf{o}_{n}$ instead of $(0,0, \ldots, 0)$ in $\mathbb{R}^{n}$.

A. Symmetric truncated hypercube. Let us consider $n+1$ hyperplanes

$$
\begin{gathered}
H_{1}=\left\{\mathbf{x} \mid-x_{1}+x_{2}+\cdots+x_{n}+1-d=0\right\}, \\
H_{2}=\left\{\mathbf{x} \mid x_{1}-x_{2}+\cdots+x_{n}+1-d=0\right\}, \\
\vdots \\
H_{n}=\left\{\mathbf{x} \mid x_{1}+x_{2}+\cdots-x_{n}+1-d=0\right\}, \\
H_{n+1}=\left\{\mathbf{x} \mid a_{1} x_{1}+a_{2} x_{2}+\cdots+a_{n} x_{n}+y=0\right\} .
\end{gathered}
$$

Then the volume is the following,

$$
\begin{aligned}
\operatorname{vol}\left([0,1]^{n} \cap H_{1}^{+} \cap \cdots \cap H_{n}^{+}\right) & =\operatorname{vol}\left([0,1]^{n} \cap H_{1}^{+} \cap \cdots \cap H_{n}^{+} \cap H_{n+1}^{+}\right)+\operatorname{vol}\left([0,1]^{n} \cap H_{1}^{+} \cap \cdots \cap H_{n}^{+} \cap H_{n+1}^{-}\right) \\
& =1-n \times \frac{d^{n}}{n !} .
\end{aligned}
$$


Electronic Journal of Linear Algebra, ISSN 1081-3810

Note that these hyperplanes do not intersect each other in $[0,1]^{n}$ under the condition $0<d<1$. Corollary 1.2 essentially suffices to compute the volume.

We can check that there are three kinds of vertices,

$$
\begin{array}{ll}
|I|=0: \quad F^{0} \backslash\left\{\mathbf{e}_{1}, \mathbf{e}_{2}, \ldots, \mathbf{e}_{n}\right\} \\
|I|=1: \quad(1-d) \mathbf{e}_{i} \quad \text { for } i=1,2, \ldots, n \\
& \mathbf{e}_{i}+d \mathbf{e}_{j} \quad \text { for } 1 \leq i \neq j \leq n .
\end{array}
$$

The resulting identity is

$$
\sum_{i=1}^{n} \frac{\left(y+a_{i}\right)^{n}}{a_{1} a_{2} \cdots a_{n}}-\sum_{i=1}^{n} \frac{\left(y+a_{i}(1-d)\right)^{n}}{a_{i} \prod_{j=1, j \neq i}^{n}\left(a_{j}+a_{i}\right)}-\sum_{1 \leq i \neq j \leq n} \frac{\left(y+a_{i}+a_{j} d\right)^{n}}{a_{j}\left(a_{i}+a_{j}\right) \prod_{t=1, t \neq i, j}^{n}\left(a_{t}-a_{j}\right)}=(-1)^{n+1} n d^{n}
$$

B. Hyperprism: $n$-simplex $\times[0,1]^{m}$. Let us consider the following two hyperplanes

$$
\begin{aligned}
& H_{1}=\left\{\mathbf{x} \mid-x_{1}-x_{2}-\cdots-x_{n}+1-\epsilon=0\right\} \\
& H_{2}=\left\{\mathbf{x} \mid a_{1} x_{1}+\cdots+a_{n} x_{n}+b_{1} x_{n+1}+\cdots+b_{m} x_{n+m}+y=0\right\} .
\end{aligned}
$$

The resulting volume taking $\epsilon \rightarrow 0$ is the following.

$$
\begin{aligned}
\operatorname{vol}\left([0,1]^{n+m} \cap H_{1}^{+}\right) & =\operatorname{vol}\left([0,1]^{n+m} \cap H_{1}^{+} \cap H_{2}^{+}\right)+\operatorname{vol}\left([0,1]^{n+m} \cap H_{1}^{+} \cap H_{2}^{-}\right) \\
& =\frac{1}{n !}
\end{aligned}
$$

We can check that there are several kinds of vertices

$$
\begin{aligned}
|I|=0: & \mathbf{o}_{n+m}, \\
& \mathbf{e}_{n+1}, \mathbf{e}_{n+2}, \ldots, \mathbf{e}_{n+m}, \\
& \mathbf{e}_{n+1}+\mathbf{e}_{n+2}, \mathbf{e}_{n+1}+\mathbf{e}_{n+3}, \ldots, \mathbf{e}_{n+m-1}+\mathbf{e}_{n+m}, \\
& \vdots \\
& \mathbf{e}_{n+1}+\mathbf{e}_{n+2}+\cdots+\mathbf{e}_{n+m},
\end{aligned}
$$

and for $i \in[n]$,

$$
\begin{aligned}
|I|=1: \quad & (1-\epsilon) \mathbf{e}_{i}+\mathbf{o}_{n+m}, \\
& (1-\epsilon) \mathbf{e}_{i}+\left\{\mathbf{e}_{n+1}, \ldots, \mathbf{e}_{n+m}\right\} \\
& (1-\epsilon) \mathbf{e}_{i}+\left\{\mathbf{e}_{n+1}+\mathbf{e}_{n+2}, \mathbf{e}_{n+1}+\mathbf{e}_{n+3}, \ldots, \mathbf{e}_{n+m-1}+\mathbf{e}_{n+m}\right\} \\
& \vdots \\
& (1-\epsilon) \mathbf{e}_{i}+\mathbf{e}_{n+1}+\mathbf{e}_{n+2}+\cdots+\mathbf{e}_{n+m} .
\end{aligned}
$$


The resulting identity is

$$
\begin{aligned}
& \sum_{I \subset\left\{b_{1}, \ldots, b_{m}\right\}}(-1)^{|I|}(y+\|I\|)^{n+m} \\
+ & \sum_{i=1}^{n} \sum_{I \subset\left\{b_{1}, \ldots, b_{m}\right\}}(-1)^{|I|+1}\left(\prod_{j=1, j \neq i}^{n} \frac{a_{j}}{a_{j}-a_{i}}\right)\left(y+a_{i}+\|I\|\right)^{n+m} \\
= & (-1)^{n+m} \frac{(n+m) !}{n !} a_{1} \cdots a_{n} b_{1} \cdots b_{m} .
\end{aligned}
$$

C. Isosceles $n$-simplex. Let us consider the following three hyperplanes

$$
\begin{aligned}
& H_{1}=\left\{\mathbf{x} \mid-x_{1}-x_{2}-\cdots-x_{n}+1-\epsilon=0\right\}, \\
& H_{2}=\left\{\mathbf{x} \mid x_{1}-x_{2}-\cdots-x_{n}-\epsilon=0\right\}, \\
& H_{3}=\left\{\mathbf{x} \mid a_{1} x_{1}+a_{2} x_{2}+\cdots+a_{n} x_{n}+y=0\right\} .
\end{aligned}
$$

The resulting volume taking $\epsilon \rightarrow 0$ is the following.

$$
\begin{aligned}
\operatorname{vol}\left([0,1]^{n} \cap H_{1}^{+} \cap H_{2}^{+}\right) & =\operatorname{vol}\left([0,1]^{n} \cap H_{1}^{+} \cap H_{2}^{+} \cap H_{3}^{+}\right)+\operatorname{vol}\left([0,1]^{n} \cap H_{1}^{+} \cap H_{2}^{+} \cap H_{3}^{-}\right) \\
& =\frac{1}{n ! 2^{n-1}} .
\end{aligned}
$$

We can check that there are two kinds of vertices

$$
\begin{aligned}
& |I|=1: \quad \epsilon \mathbf{e}_{1},(1-\epsilon) \mathbf{e}_{1}, \\
& |I|=2: \quad \frac{1}{2} \mathbf{e}_{1}+\left(\frac{1}{2}-\epsilon\right) \mathbf{e}_{i} \quad \text { for } \quad 2 \leq i \leq n
\end{aligned}
$$

This case needs Corollary 5.1 for the three hyperplane case.

The resulting identity is

$$
\begin{aligned}
& \frac{y^{n}}{a_{1}\left(a_{2}+a_{1}\right)\left(a_{3}+a_{1}\right) \cdots\left(a_{n}+a_{1}\right)}-\frac{\left(y+a_{1}\right)^{n}}{a_{1}\left(a_{2}-a_{1}\right)\left(a_{3}-a_{1}\right) \cdots\left(a_{n}-a_{1}\right)} \\
& -2 \sum_{i=2}^{n} \frac{\left(y+\frac{a_{1}}{2}+\frac{a_{i}}{2}\right)^{n}}{\left(a_{1}+a_{i}\right)\left(a_{1}-a_{i}\right)\left(a_{2}-a_{i}\right) \cdots\left(a_{n}-a_{i}\right)}=(-1)^{n} 2^{1-n} .
\end{aligned}
$$

D. Trapezoidal polytope. Let us consider the following two hyperplanes

$$
\begin{aligned}
& H_{1}=\left\{\mathbf{x} \mid-\frac{x_{1}}{2}-\frac{x_{2}}{2}-\cdots--\frac{x_{n}}{2}-x_{n+1}-x_{n+2}-\cdots-x_{n+m}+1-\epsilon=0\right\}, \\
& H_{2}=\left\{\mathbf{x} \mid a_{1} x_{1}+\cdots+a_{n} x_{n}+b_{1} x_{n+1}+\cdots+b_{m} x_{n+m}+y=0\right\} .
\end{aligned}
$$

The resulting volume taking $\epsilon \rightarrow 0$ is the following.

$$
\begin{aligned}
\operatorname{vol}\left([0,1]^{n+m} \cap H_{1}^{+}\right) & =\operatorname{vol}\left([0,1]^{n+m} \cap H_{1}^{+} \cap H_{2}^{+}\right)+\operatorname{vol}\left([0,1]^{n+m} \cap H_{1}^{+} \cap H_{2}^{-}\right) \\
& =\frac{2^{n}-n 2^{-m}}{(n+m) !} .
\end{aligned}
$$


We can check that there are four kinds of vertices:

$$
\begin{array}{ll}
|I|=0: \quad & \mathbf{o}_{n+m}, \mathbf{e}_{1}, \mathbf{e}_{2}, \ldots, \mathbf{e}_{n}, \\
|I|=1: \quad & (1-\epsilon) \mathbf{e}_{n+i} \quad \text { for } 1 \leq i \leq m, \\
& \mathbf{e}_{i}+\left(\frac{1}{2}-\epsilon\right) \mathbf{e}_{n+j} \quad \text { for } 1 \leq i \leq n, 1 \leq j \leq m, \\
& \mathbf{e}_{i}+(1-2 \epsilon) \mathbf{e}_{j} \text { for } 1 \leq i \neq j \leq n .
\end{array}
$$

The resulting identity is

$$
\begin{aligned}
& \frac{(-1)^{n+m} y^{n+m}}{a_{1} \cdots a_{n} b_{1} \cdots b_{m}}+\sum_{i=1}^{n} \frac{(-1)^{n+m-1}\left(y+a_{i}\right)^{n+m}}{a_{1} \cdots a_{n} b_{1} \cdots b_{m}}+\sum_{j=1}^{m} \frac{\left(y+b_{j}\right)^{n+m}}{b_{j} \prod_{s=1}^{n}\left(\frac{b_{j}}{2}-a_{s}\right) \prod_{t=1, t \neq j}^{m}\left(b_{j}-b_{t}\right)} \\
& -\sum_{i=1}^{n} \sum_{j=1}^{m} \frac{\left(y+a_{i}+\frac{b_{j}}{2}\right)^{n+m}}{b_{j} \prod_{s=1}^{n}\left(\frac{b_{j}}{2}-a_{s}\right) \prod_{t=1, t \neq j}^{m}\left(b_{j}-b_{t}\right)}-\sum_{1 \leq i \neq j \leq n} \frac{\left(y+a_{i}+a_{j}\right)^{n+m}}{2^{m} a_{i} \prod_{s=1, s \neq i}^{n}\left(a_{i}-a_{s}\right) \prod_{t=1}^{m}\left(a_{i}-\frac{b_{t}}{2}\right)} \\
& =2^{n}-n 2^{-m} .
\end{aligned}
$$

Acknowledgments. The authors would like to thank Professor Eungchun Cho at Kentucky State University for introducing this problem, and Gabriel C. Drummond-Cole for his helpful comments. The authors appreciate several anonymous referees for many valuable suggestions and corrections.

\section{REFERENCES}

[1] D.L. Barrow and P.W. Smith. Spline notation applied to a volume problem. The American Mathematical Monthly, 86:50-51, 1979.

[2] E. Cho and Y. Cho. The volume of simplices clipped by a half space. Applied Mathematics Letters, 14:731-735, 2001.

[3] E. Cho and Y. Cho. An exact and explicit method for volume of hypercubes clipped by two or three hyperplanes. Preprint.

[4] M.E. Dyer and A.M. Frieze. On the complexity of computing the volume of a polyhedron. SIAM Journal on Computing, 17:967-974, 1988.

[5] P. Filliman. The volume of duals and sections of polytopes. Mathematika. A Journal of Pure and Applied Mathematics, 39:67-80, 1992.

[6] P. Gritzmann and V. Klee. On the complexity of some basic problems in computational convexity. II. Volume and mixed volumes. Polytopes: Abstract, convex and computational, Proceedings of the NATO Advanced Study Institute held in Scarborough, 440:373-466, 1994.

[7] L.G. Khachiyan. The problem of calculating the volume of a polyhedron is enumerably hard. Russian Mathematical Surveys, 44:199-200, 1989.

[8] J.B. Lasserre. An analytical expression and an algorithm for the volume of a convex polyhedron in $\mathbb{R}^{n}$. Journal of Optimization Theory and Applications, 39:363-377, 1983.

[9] J. Lawrence. Polytope volume computation. Mathematics of Computation, 57:259-271, 1991.

[10] D.H. Lehmer. The Tarry-Escott problem. Scripta Mathematica, 13:37-41, 1947.

[11] J.-L. Marichal and M.J. Mossinghoff. Slices, slabs, and sections of the unit hypercube. Online Journal of Analytic Combinatorics, 3:1-11, 2008.

[12] G. Polya. Berechnung eines bestimmten integrals. Mathematische Annalen, 74:204-212, 1913.

[13] S.M. Ruiz. An algebraic identity leading to Wilson's theorem. The Mathematical Gazette, 80:579-582, 1996.

[14] C. Zong. What is known about unit cubes. Bulletin of the American Mathematical Society, 42:181-211, 2005. 